\title{
Lack of food intake during shift work alters the heart transcriptome and leads to cardiac tissue fibrosis and inflammation in rats
}

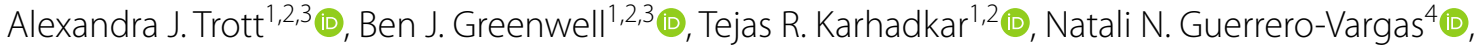 \\ Carolina Escobar ${ }^{4}$ (D) Ruud M. Buijs ${ }^{5}$ (i) and Jerome S. Menet ${ }^{1,2,3^{*}}$
}

\begin{abstract}
Background: Many epidemiological studies revealed that shift work is associated with an increased risk of a number of pathologies, including cardiovascular diseases. An experimental model of shift work in rats has additionally been shown to recapitulate aspects of metabolic disorders observed in human shift workers, including increased fat content and impaired glucose tolerance, and used to demonstrate that restricting food consumption outside working hours prevents shift work-associated obesity and metabolic disturbance. However, the way distinct shift work parameters, such as type of work, quantity, and duration, affect cardiovascular function and the underlying mechanisms, remains poorly understood. Here, we used the rat as a model to characterize the effects of shift work in the heart and determine whether they can be modulated by restricting food intake during the normal active phase.

Results: We show that experimental shift work reprograms the heart cycling transcriptome independently of food consumption. While phases of rhythmic gene expression are distributed across the 24-h day in control rats, they are clustered towards discrete times in shift workers. Additionally, preventing food intake during shift work affects the expression level of hundreds of genes in the heart, including genes encoding components of the extracellular matrix and inflammatory markers found in transcriptional signatures associated with pressure overload and cardiac hypertrophy. Consistent with this, the heart of shift worker rats not eating during work hours, but having access to food outside of shift work, exhibits increased collagen 1 deposition and displays increased infiltration by immune cells. While maintaining food access during shift work has less effects on gene expression, genes found in transcriptional signatures of cardiac hypertrophy remain affected, and the heart of shift worker rats exhibits fibrosis without inflammation.
\end{abstract}

Conclusions: Together, our findings unraveled differential effects of food consumption on remodeled transcriptional profiles of the heart in shift worker rats. They also provide insights into how shift work affects cardiac function and suggest that some interventions aiming at mitigating metabolic disorders in shift workers may have adverse effects on cardiovascular diseases.

Keywords: Shift work, Circadian rhythms, Heart, Transcriptome, RNA-Seq, Cardiac fibrosis, Inflammation, Cardiovascular diseases

\footnotetext{
*Correspondence: menet@bio.tamu.edu

${ }^{1}$ Department of Biology, Texas A\&M University, College Station, TX 77843, USA

Full list of author information is available at the end of the article
}

\section{Background}

Shift work is an employment practice designed to provide services outside the daytime working hours, including evening or night shifts, early morning shifts, and rotating shifts. Shift work is becoming increasingly popular in modern society, mostly because of the higher demand 
for productivity [1]. About $15-20 \%$ of the workforce is engaged in some kind of shift work worldwide, and this number is rapidly increasing [1]. Over the last decades, many epidemiological studies revealed that shift work affects human health and increases the risk of developing cancer, metabolic disorders, cardiovascular diseases, and mood disorders [1-14]. Relevant to this paper, a metaanalysis performed on 17 studies concluded that shift workers exhibit a $40 \%$ increased risk of developing cardiovascular diseases than day workers [15]. A more recent meta-analysis on 173,000 individuals similarly found that shift/night workers have an increased risk of cardiovascular diseases than day workers [13]. However, there was significant heterogeneity between studies considered in these meta-analyses, and it is still unclear to which extent the quantity, the duration, and the type of shift work impair cardiovascular functions $[10,12]$.

While the mechanisms by which shift work causes cardiovascular diseases remain unclear, they likely involve a misalignment between internal biological rhythms and the natural light to dark cycle [16]. In mammals, every tissue harbors a timekeeping mechanism, the circadian clock, that drives rhythms in gene expression to allow biological functions to perform optimally at the most appropriate time of the day. Circadian clocks in peripheral tissues are synchronized to the daily environmental variation by the master circadian pacemaker located in the suprachiasmatic nucleus $(\mathrm{SCN})$ of the hypothalamus, which is itself entrained to the light to dark cycle via direct retinal innervation [17]. The SCN utilizes multiple cues to synchronize peripheral clocks, including rhythms in neuronal signaling, hormone secretion, body temperature, and food intake. Alteration in the timing of these cues by shift work disrupts the proper functioning of peripheral clocks and clock-controlled biological functions. Accumulating evidence indicates that alteration of the daily rhythm of food intake, which is prevalent in shift workers, is particularly associated with circadian rhythm disorders $[18,19]$. For example, rats subjected to shift work paradigms for 5 weeks shift their food intake to the work phase (i.e., natural rest phase) and exhibit increased fat content and impaired glucose tolerance [20, 21]. Importantly, these effects are not observed if rats cannot eat during work, indicating that the adverse effects of shift work on metabolic functions are mostly mediated by a shift in the timing of food intake to the natural rest phase [20,21]. Whether rats subjected to shift work also develop cardiovascular diseases, and whether those can be prevented by restricting food intake during the normal active phase, remain however unexplored.

To get insights into how shift work alters heart biology, we used a model of shift work in rats that mimics shift work in humans [22]. Rats were housed in a slowly rotating wheel for $8 \mathrm{~h}$ during their sleeping phase from Monday to Friday and left undisturbed outside their work schedule. Because restricting food intake to the normal active phase prevents the development of metabolic disorders $[20,21]$, we hypothesized that preventing access to food during the daily 8 hours of shift work might also hinder alterations of cardiac functions. Our results indicate that five weeks of shift work in rats leads to cardiac fibrosis and that the adverse effects of shift work in the heart are surprisingly exacerbated when shift worker rats do not have access to food during work. Our findings thus suggest that lack of food intake during shift work, an intervention that prevents metabolic disorders in shift workers, has adverse effects on cardiovascular functions.

\section{Results}

To characterize the effects of shift work in the heart, we used a rat model of shift work previously shown to cause metabolic disorders similar to those observed in human shift workers [20, 22]. Because restricting food access to the natural active phase prevents the development of obesity and diabetes in this model, we also aimed to investigate the effects of lack of food intake during work (Fig. 1) [20]. A total of 54 rats maintained on a 12:12 light to dark cycle (LD12:12, light on from 7 am to $7 \mathrm{pm}$ ) were randomly assigned to control (C), shift work (W), or shift work with restricted nighttime feeding (WRF) groups prior the start of the experiment. Shift work consisted of rats being placed in a slowly rotating drum (1 revolution per $3 \mathrm{~min}$ ) from 9 am to $5 \mathrm{pm}$, Monday to Friday, for a total of 5 weeks (Fig. 1), as published previously [20, 22]. Control rats had access to food and water ad libitum throughout the experiment. Worker rats had access to ad libitum food and water and were exposed to shift work for 5 weeks. WRF rats were also exposed to shift work and had ad libitum access to water, but only had access to food when not doing shift work (i.e., from $5 \mathrm{pm}$ to $9 \mathrm{am}$; Fig. 1). For each group, rats were left undisturbed and had free access to food and water during weekends. After 5 weeks of shift work, rats were euthanized on a Saturday (weekend schedule, i.e., no work), and the hearts collected and flash-frozen.

\section{Shift work reprograms the heart cycling transcriptome}

To determine the effects of shift work in the heart, we first performed an RNA-Seq analysis of the heart transcriptome across the 24-h day in C, W, and WRF rats (6 timepoints, $n=3$ biological replicates per timepoint and group). Analysis of rhythmic gene expression revealed large differences in the number of rhythmically expressed genes, with the number of rhythmic genes being $\sim 2$-fold higher in WRF rats than in $C$ rats, and $\sim 2$-fold lower in $\mathrm{W}$ rats than in $\mathrm{C}$ rats (Fig. 2A, Additional file 1: Table S1). 


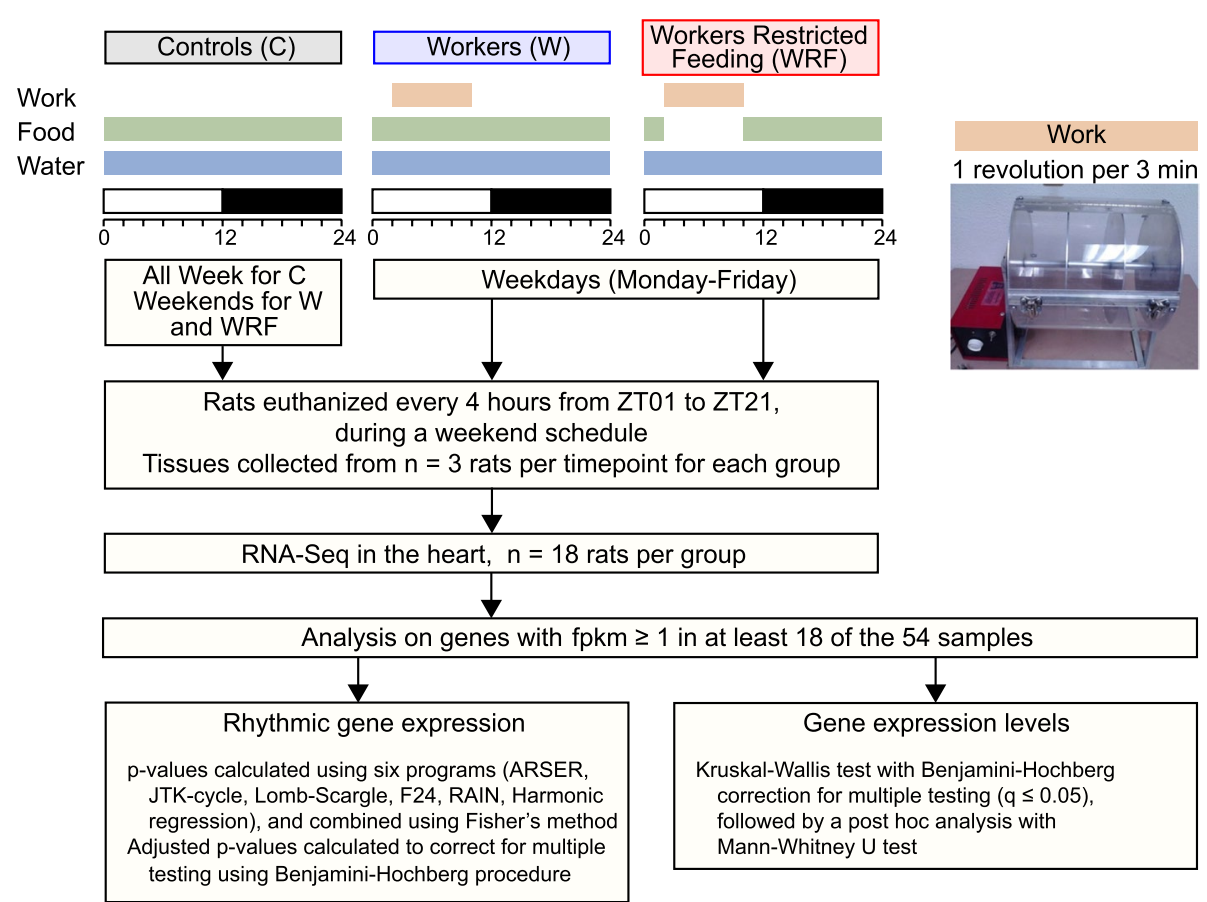

Fig. 1 Experimental setup. Individually housed rats exposed to LD12:12 were randomly assigned to one of the three groups: control (C), shift worker (W), or shift worker subjected to restricted feeding during work (WRF). All rats had ad libitum access to water. $C$ rats had ad libitum access to food and were not manipulated throughout the experiment. W and WRF rats were placed in a rotating drum (1 revolution per $3 \mathrm{~min}$ ) for $8 \mathrm{~h}$ (9 am-5 pm, i.e., ZT2 to ZT10), Monday-to-Friday, for 5 consecutive weeks to simulate shift work, and were maintained unrestrained in their cages over the weekend. W rats had ad libitum access to food at all times, including during work, whereas WRF rats only had access to food outside the work schedule, i.e., from $5 \mathrm{pm}$ to $9 \mathrm{am}$. After 5 weeks, rats were euthanized during a week-end schedule (no work) every $4 \mathrm{~h}$ with $n=3$ rats per timepoint and group. Hearts were collected, flash-frozen, and processed afterward for RNA extraction and RNA-Seq library preparation

Similar results were obtained with a differential rhythmicity analysis using dryR (Additional file 1: Table S2, Additional file 2: Fig. S1) [23]. Rhythmically expressed genes poorly overlapped between the three groups, suggesting that shift work profoundly reprograms the rat heart cycling transcriptome (Fig. 2A, B). Interestingly, while phases of rhythmic gene expression in $C$ rats were distributed across the 24-h day with most genes peaking between ZT0 and ZT20, rhythmic genes in both W and WRF rats displayed discrete peak phases of expression, i.e., ZT11-14 for W rats, and ZT7-9 and ZT20-21 in WRF rats (Fig. 2B, C). This suggests that shift work, which occurs recurrently from ZT2 to ZT10, stimulates gene expression at specific phases of the day, and that this peak of expression persists the day following shift work when animals are left undisturbed, i.e., when heart samples were collected on a Saturday (Fig. 2A).

Examination of core clock gene expression showed that shift work alone impaired the molecular clock oscillation in the heart and that preventing food access during shift work restored rhythmic gene expression (Fig. 2D). However, the amplitude of rhythmic expression in WRF rats was significantly decreased for most clock genes (Fig. 2D,
Additional file 2: Fig. S2A). Moreover, arrhythmic expression of clock genes in $\mathrm{W}$ heart was mostly due to differences in peak expression between biological replicates, with the replicate rhythm 2 showing out-of-phase rhythmic expression compared to the replicate rhythms 1 and 3 (Additional file 2: Fig. S2B). While it remains unclear why the molecular clock oscillation is shifted in only one replicate rhythm, it is possible that the heart molecular clock is less sensitive to shift work synchronizing cues (e.g., increased daytime food intake) than other tissues like the liver (see the "Discussion" section) [20, 22].

To determine if shift work reprograms the rhythmicity of specific biological pathways, we performed a KEGG pathway analysis [24] of genes being rhythmically expressed in either C, W, or WRF rats. Consistent with our finding that rhythmic genes poorly overlap between groups, most pathways were enriched in a group-specific manner (Fig. 2E, Additional file 1: Table S3). Genes being rhythmically expressed in $\mathrm{C}$ rats were enriched for various signaling pathways, including MAPK, TNF, p53, and FoxO signaling pathways (Fig. 2E). Although rhythmic genes in W and WRF rats were both enriched in metabolic pathways, the nature of these pathways was 


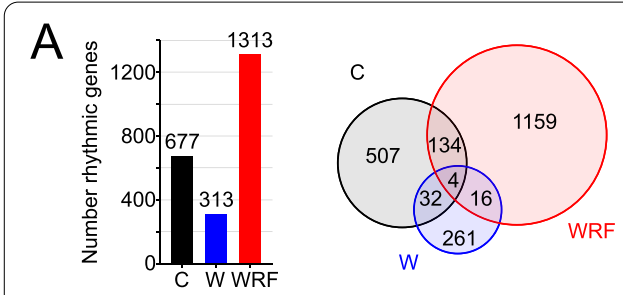

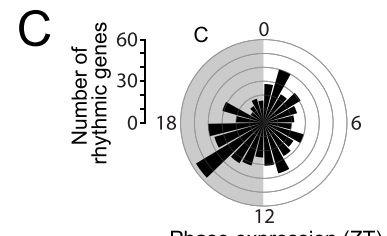

Phase expression (ZT)

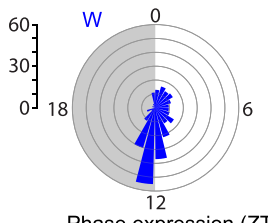

Phase expression (ZT)

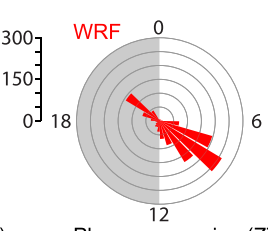

Phase expression (ZT)
B
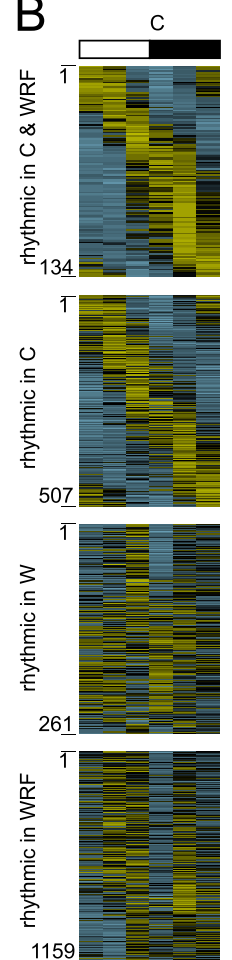

W
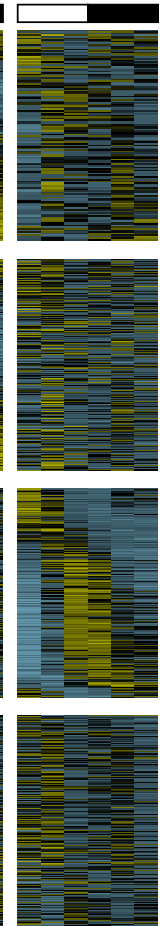

z-score
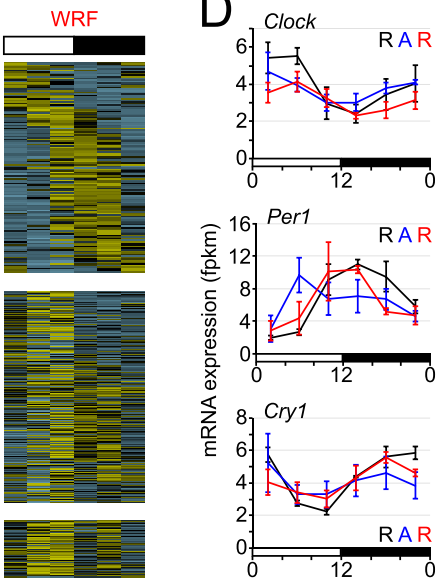

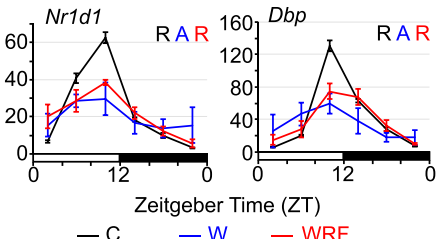

E

Circadian rhythm

MAPK signaling pathway

Pathways in cancer

TNF signaling pathway

Notch signaling pathway

p53 signaling pathway

FoxO signaling pathway

FoxO signa

Apoptosis
Hippo signaling pathway

Steroid binaling pathway

Ras signaling pathway

Ras signaling pathway
Pyrimidine metabolism

Folate biosynthesis

Carbon metabolism

Citrate cycle (TCA cycle)

Biosynthesis of amino acids

Metabolic pathways

Aminoacyl-tRNA biosynthesis

Peroxisome

$A B C$ transporters

AMPK signaling pathway

Spliceosome

Adipocytokine signaling pathway

Nitrogen metabolism

Adrenergic signal. in cardiomyocytes

Oxidative phosphorylation

Thermogenesis

Non-alcoholic fatty liver disease

Amyotrophic lateral sclerosis

Adherens junction

Protein processing in ER

Cardiac muscle contraction

Cell cycle

Platelet activation

Wnt signaling pathway

TGF-beta signaling pathway

Lysosome

Autophagy - animal

HIF-1 signaling pathway

Dilated cardiomyonathy

Regulation of actin cytoskeleton

Sphingolipid metabolism

Thyroid hormone signaling pathway

Toll-like receptor signaling pathway

Enrichment $(-\log 10)$
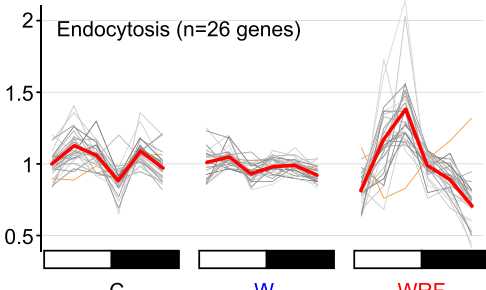

$\mathrm{C}$

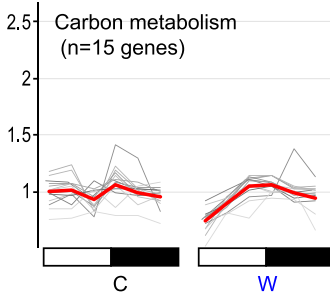

Rhythmic in

C W WRF
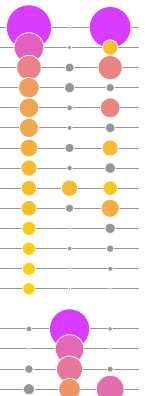

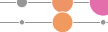

- - - - - -

$-$

$\ldots \ldots$

$\longrightarrow-$

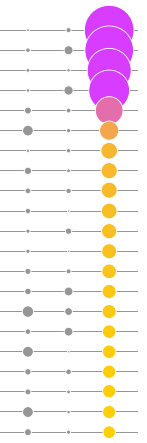

F

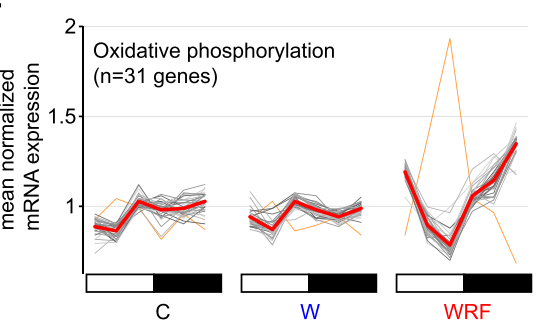

Fig. 2 Effect of shift work on the cycling heart transcriptome. A Total number (left) and overlap (right) between rhythmically expressed genes for each group: control (C, black), shift worker (W, blue), or shift worker subjected to restricted feeding during work (WRF, red). B Heatmap visualization of rhythmic gene expression for genes rhythmic in both C and WRF groups, C only, W only, and WRF only. The signal for each timepoint corresponds to the average of 3 individual samples. Genes were ordered based on the phase of rhythmic gene expression of $C$ rats for the top two heatmaps, while they were ordered based on the phase of rhythmic gene expression in W and WRF groups for genes rhythmic in W only and WRF only, respectively. C Rose plot representation of the phase of rhythmic gene expression in C, W, and WRF rat groups. D Clock gene rhythmic expression in the heart of $C, W$, and WRF rats. The signal corresponds to the mean + /- s.e.m of 3 animals per timepoint. Statistical analysis for rhythmic gene expression is displayed as R (rhythmic expression) or A (arrhythmic expression), and the font color refers to each group: C, black; W, blue; WRF, red. E KEGG pathway enrichment for genes being rhythmically expressed in C,W, and WRF rat heart. Pathways that are not enriched in certain groups $(p>0.05)$ are displayed in grey. Diameter of each circle is also proportional to the enrichment $q$ value, with higher diameter for lower $q$ values. $\mathbf{F}$ Expression profile of genes accounting for the enrichment of three KEGG pathways. The expression of individual genes is displayed in gray and the averaged expression in red. For the oxidative phosphorylation and endocytosis pathways (WRF enriched pathways), the rhythmic expression of one gene is in antiphase to all other genes; this gene is displayed in orange and was not taken into account for calculating the averaged expression 
group-specific, likely reflecting differences in the timing of food intake between W and WRF rats [20]. Rhythmic genes in $\mathrm{W}$ rats were enriched in pathways involved in carbon metabolism, biosynthesis of amino acid and aminoacyl tRNA, and nitrogen metabolism, whereas rhythmic genes in WRF group were mostly involved in oxidative phosphorylation (Fig. 2E). Genes being rhythmically expressed in WRF rats were also enriched in pathways associated with membrane-enclosed organelles (e.g., protein processing in the endoplasmic reticulum, lysosome, endocytosis, and autophagy), as well as pathways associated with cardiac functions such as cardiac muscle contraction and dilated cardiopathy (Fig. 2E).

Given that most rhythmic genes in W and WRF rats peaked at discrete phases (Fig. 2C), we examined the expression of rhythmic genes from several enriched KEGG pathways (Fig. 2F). Remarkably, rhythmic genes from the same enriched pathways displayed similar patterns of expression (Fig. 2F, Additional file 1: Table S4, Additional file 2: Fig. S3). For example, the expression of 30 out of 31 rhythmic genes involved in oxidative phosphorylation was highly coordinated in WRF rats, with trough expression at ZT9 at the end of shift work. Similarly, 25 out of 26 rhythmic genes involved in endocytosis displayed similar expression profiles in WRF rats, with peak expression at the end of shift work. Moreover, 15 rhythmic genes in W rats involved in carbon metabolism showed similar profiles of expression and peaking a few hours after the end of shift work.

Taken together, our data indicate that both shift work and shift work without food intake profoundly reprogram the heart cycling transcriptome. Many genes normally expressed rhythmically in rat heart across the 24-h day lose rhythmicity. Conversely, both shift work protocols lead to the emergence of new rhythms of gene expression, which for the most part occur at discrete phase across the day and are likely to be directly induced by the lack of food intake during shift work or by shift work alone.

\section{Gene expression in the heart is profoundly altered in shift workers subjected to restricted feeding}

To further investigate the effects of shift work in the heart, we also performed an analysis of differential gene expression between the 3 groups (Fig. 1). This analysis revealed that 944 genes (9.2\% of all expressed genes) were affected by shift work (Fig. 3A, Additional file 1: Table S5, Additional file 2: Fig. S4). Remarkably, the misregulation of heart gene expression by shift work was almost exclusively restricted to the WRF group ( $n$ $=904$ genes; $\mathrm{W}, n=40$ genes) (Fig. 3A). In particular, the expression of 808 genes were significantly increased in WRF rats when compared to $C$ and W rats, while only 67 genes exhibited a decreased expression (Fig. 3B).

Visualization of altered gene expression with heatmaps revealed biological variation between rats from the same group, indicating that rats were not equally affected by the different shift work protocols (Fig. 3C). This variation was not time-dependent, as strong effects of WRF protocol were observed for heart samples collected at every time point. Rather, the effects on gene expression appeared to be individual-specific, with individuals affected by shift work displaying a homogenous transcriptional response to shift work, i.e., the impact of shift work on gene expression was observed for most genes (Fig. 3C). This suggests that shift work in WRF rats impacts entire transcriptional programs rather than random genes and that some key master transcriptional regulators are affected by the lack of food intake during shift work.

To get insights into the transcriptional programs and biological pathways that were affected in the heart of WRF rats, we performed a KEGG pathway analysis using the 808 and 67 genes that were upregulated and downregulated in WRF rats, respectively. This analysis revealed a significant upregulation of pathways associated with gene expression, including ribosome biogenesis, proteasome, spliceosome, and protein processing in the endoplasmic reticulum (Fig. 3D). In particular, 73 out of the 75 ribosomal protein genes that constitute the eukaryotic $80 \mathrm{~S}$ ribosome showed increased expression in WRF rats (Fig. 3E). Similarly, 26 out of the 34 genes that make up the eukaryotic $26 \mathrm{~S}$ proteasome displayed increased expression in WRF rats (Fig. 3F). Interestingly, genes upregulated in WRF were also enriched for pathways associated with the cell cycle, such as DNA replication, nucleotide excision repair, mismatch repair, and purine and pyrimidine metabolism. Together, these data suggest that changes in gene expression in the heart of WRF rats are associated with increased gene expression and cell division, a feature that may be related to cardiac hypertrophy as suggested in other studies [25-27].

The KEGG pathway analysis also revealed that genes exhibiting decreased expression in WRF rats were associated with various signaling pathways, including cholinergic synapse and adrenergic signaling in cardiomyocytes (Fig. 3D). While it is unclear why various signaling pathways are enriched for decreased gene expression in the heart of WRF rats, this may reflect the conflicting information between activity (i.e., work) and lack of food intake that WRF rats experienced. 


\begin{tabular}{|c|c|}
\hline$A$ & Number of genes \\
\hline $\begin{array}{l}\text { Genes expressed } \\
\text { in the rat heart }\end{array}$ & $n=10,257$ \\
\hline $\begin{array}{l}\text { Genes significantly } \\
\text { affected by W }\end{array}$ & $n=40(0.39 \%)$ \\
\hline $\begin{array}{l}\text { Genes significantly } \\
\text { affected by WRF }\end{array}$ & $\mathrm{n}=904(8.81 \%)$ \\
\hline
\end{tabular}
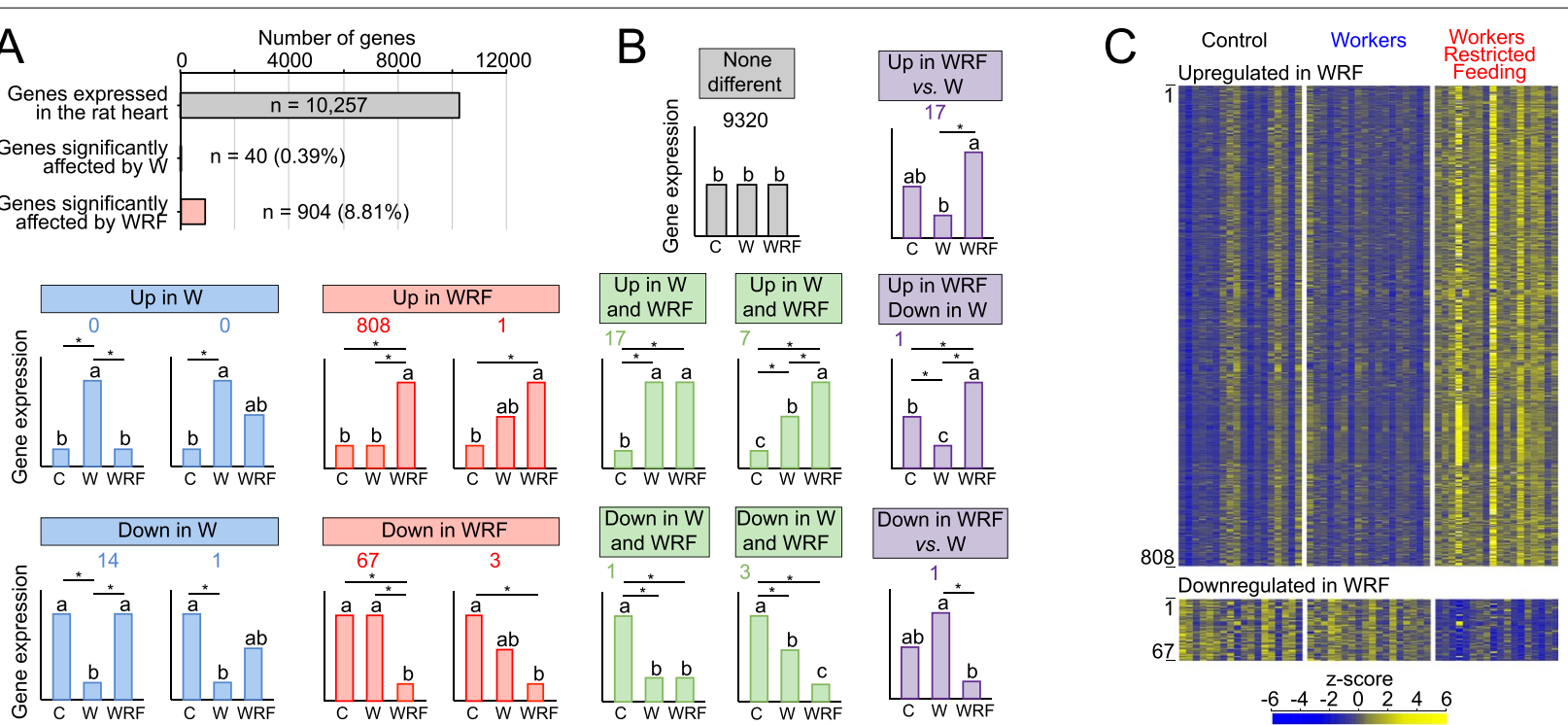
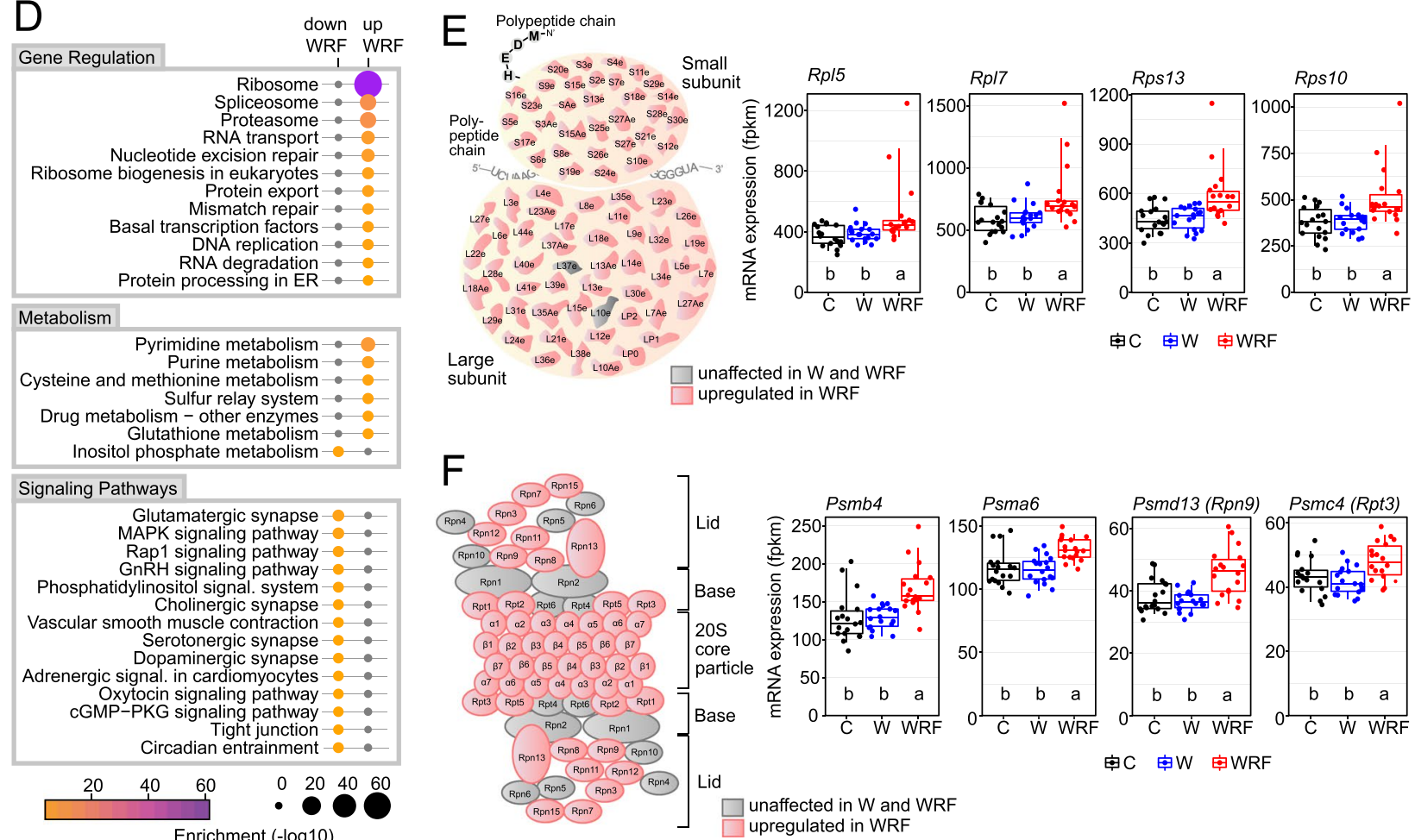

白C 白W 自WRF
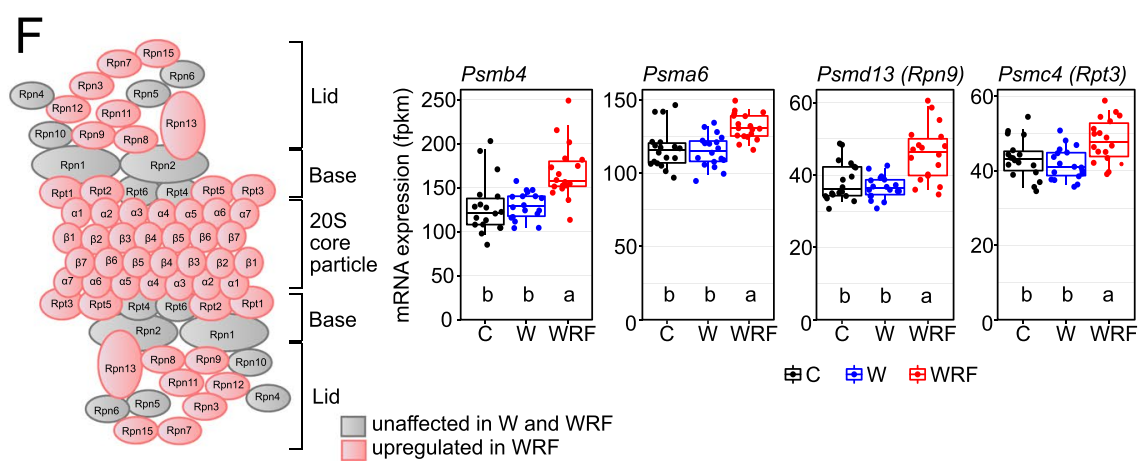

由 自W 白WRF

Fig. 3 Effect of shift work on cardiac gene expression. A, B Number of differentially expressed genes between C, W, and WRF groups (A), and description of the significant effects between groups after Mann-Whitney $U$ test post hoc analysis (B). Genes were considered differentially expressed between groups if $q$ value $\leq 0.05$. Categories of statistically significant differential gene expression are illustrated by bar graphs with the total number of genes written above for each category. Groups with different letters are significantly different. The majority of the differentially expressed genes were either upregulated $(n=808)$ or downregulated $(n=67)$ in WRF compared to C and W. C Heatmap of standardized gene expression for genes upregulated or downregulated in WRF rats. The 18 animals used for each paradigm are sorted by the time of euthanasia. Higher levels of gene expression are displayed in yellow. D Gene Ontology and KEGG pathways analysis of genes being significantly upregulated in the heart of WRF rats $(n=808)$. $\mathbf{E}$, $\mathbf{F}$ Left: Illustration of the $80 S$ ribosome $(\mathbf{E})$ and 265 proteasome $(\mathbf{F})$, with upregulated genes in WRF rats vs. $C$ and W rats being displayed in red. Genes whose expression is unaffected are displayed in gray. Right: expression profiles of ribosomal genes (E) and proteasomal genes (F) being upregulated in WRF rats. Each dot represents individual fpkm values. Groups with different letters are significantly different $(p<0.05$, Kruskal-Wallis test) 


\section{Altered gene expression in the heart of shift workers subjected to restricted feeding is associated} with transcription factors known to regulate heart biology The homogenous change in gene expression that was observed in each WRF rat (Fig. 3C) suggests that transcriptional programs driven by specific transcription factors are altered. Findings by the ENCODE consortium and others revealed that $\sim 95 \%$ of transcription factors bind in open chromatin regions, i.e., in genomic regions that are more sensitive to mild nuclease digestion [2830]. To get insights into the mechanisms underlying differential gene expression between WRF vs. C and W rats, we set out to uncover open chromatin regions in rat heart by carrying out DNase-Seq to identify the transcription factors that may be involved.

Analysis of our rat heart DNase-Seq dataset revealed that 28,514 genomic regions were more accessible to DNase I digestion, with a vast majority being located as expected in intergenic regions, transcription start sites, and introns (Fig. 4A, Additional file 1: Table S6). To further assess the quality of our dataset, we performed a cross-comparison of heart DNase-Seq peaks between rat and mouse using mouse heart DNase-Seq datasets from the mouse ENCODE project [28]. Using stringent peak calling, we found that that $71.2 \%$ of the rat DNase-Seq peaks overlapped with mouse DNase-Seq peaks $(15,480$ peaks out of 21,750 peaks) (Fig. 4B). Importantly, visualization of rat and mouse DNase-Seq profiles over syntenic genomic regions confirmed that many DNase hypersensitive sites (DHS, i.e., open chromatin regions) are conserved between rat and mouse, thereby validating our rat heart DNase-Seq dataset for further analysis of the transcription factors that contribute to the changes in gene expression in WRF rats (Fig. 4C).

To identify these transcription factors, we conducted a motif analysis using HOMER in the open chromatin regions located within the 808 and 67 genes that were significantly increased and decreased in WRF rats, respectively [31]. Several consensus motifs were identified, including those bound by the transcription factors belonging to the ETS family, the KLF family, NRF family, bHLH family, and for Ronin (Fig. 4D, Additional file 1: Table S7). Interestingly, many of these transcription factors have been involved in the development of cardiovascular disorders and cardiac fibrosis (Table 1). For example, the ETS transcription factor Ets1 has been shown to mediate angiotensin II-related cardiac fibrosis and cardiac hypertrophy [32, 33], while increased ETV1 activity has been shown to induce atrial remodeling and arrhythmia [34]. Moreover, the SP/KLF family transcription factors $K l f 3$ and Klf6 have been involved in cardiovascular development and associated with cardiac fibrosis, respectively $[35,36]$. In addition, the IRF family transcription factor IRF8 has been shown to suppress pathological cardiac remodeling by inhibiting calcineurin signaling [37] (Table 1).

Visualization of the transcript levels for these transcription factors in the heart of W, WRF, and $C$ rats revealed that shift work protocols did not dramatically alter their expression (Fig. 4E). Only two of them, Elk1 and Erg, showed decreased expression in W and WRF rats when compared to $\mathrm{C}$ rats (Fig. $4 \mathrm{E}$ ). While we cannot exclude that the misregulation of gene expression in WRF rats is solely caused by Erg decreased expression in WRF rat heart, our data suggest that the lack of food intake during shift work alters heart gene expression by impairing the activity of several transcription factors (e.g., through mistimed activation of signaling pathways) rather than by affecting the expression level of transcription factors.

We also leveraged our rat heart DNase-Seq dataset to investigate transcription factor motifs enriched in DHSs located in the loci of genes rhythmic in either $\mathrm{C}, \mathrm{W}$, or WRF rat hearts. While e-box motif was enriched in open chromatin regions of rhythmic genes in $\mathrm{C}$ and WRF rat hearts (and to a lesser extent in W rat hearts), glucocorticoid receptor motif was found to be only enriched for rhythmic genes in $\mathrm{C}$ rats (Additional file 1: Table S8, Additional file 2: Fig. S5). Importantly, motifs for the transcription factors belonging to the ETS family and KLF family were strongly enriched in genes being rhythmic in WRF rat hearts, but not in $\mathrm{C}$ and $\mathrm{W}$ rats (Additional file 1: Table S8, Additional file 2: Fig. S5), thus suggesting that their increased activity in WRF rats contributes to the rhythmic expression of their target genes. Together, these data strongly suggest that differences of transcription factor activity during shift work underlie the differences in rhythmic gene expression in rat heart.

\section{Genes whose expression is altered by shift work are associated with cardiac diseases}

Several studies have characterized transcriptional signatures of cardiovascular disorders in both mice and humans. To determine whether genes being altered by shift work are also misregulated in pathological heart samples, we first examined a paper that identified cardiomyocyte gene programs being activated after cardiac hypertrophy and heart failure in the mouse [25]. This study relied on transverse aorta constriction to promote pressure overload and induce cardiac hypertrophy 1-2 weeks after surgery and heart failure after 4-8 weeks. Using RNA-Seq of cardiomyocytes collected from 3 days to 8 weeks post-surgery, the authors clustered genes into 56 cardiac hypertrophy stage-specific modules that characterized distinct gene programs encoding morphological and functional transcriptional signatures occurring at 

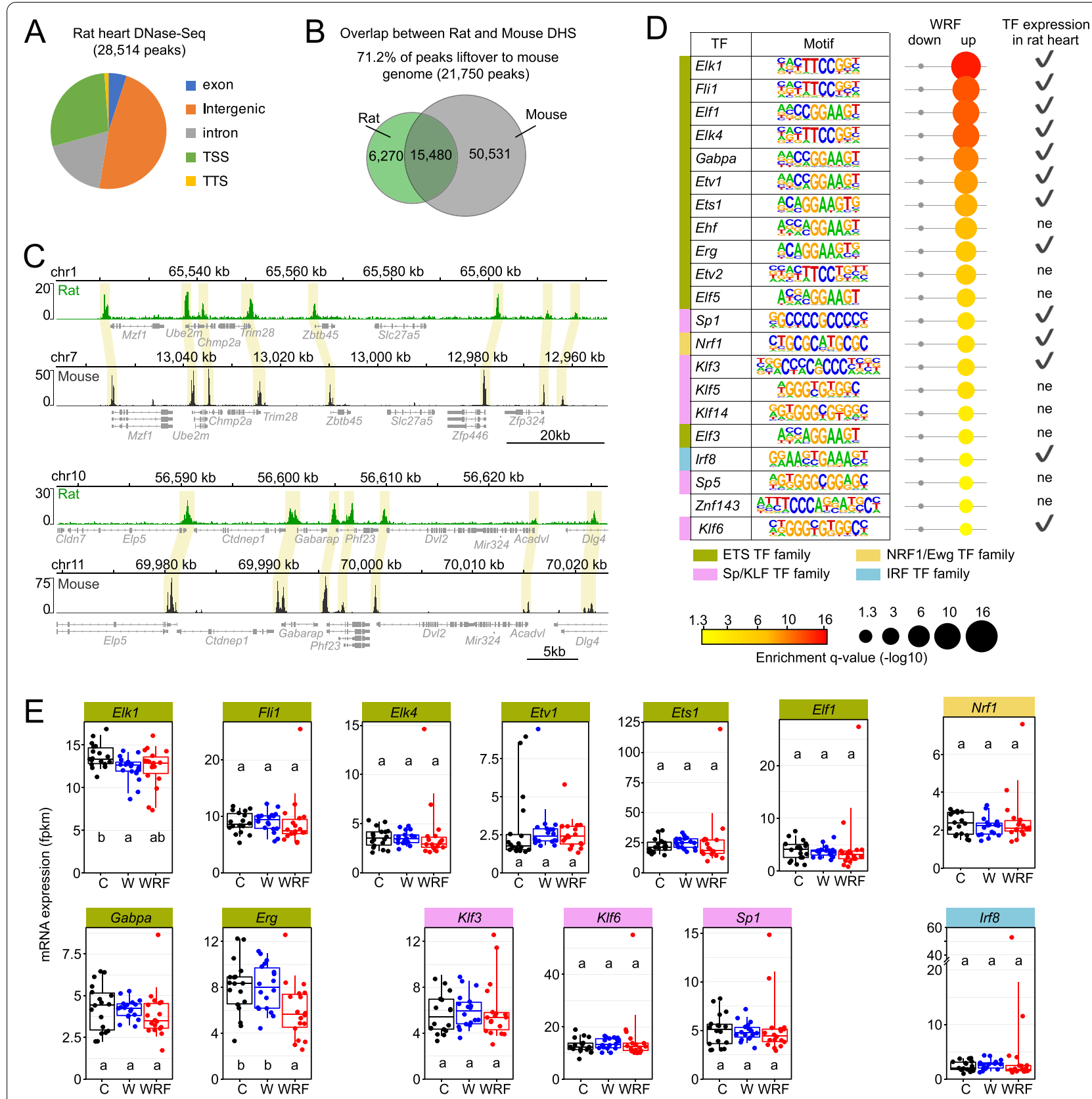

Fig. 4 Transcription factor motif analysis at genes being misregulated in the heart of WRF rats. A Genomic location of DNase I hypersensitive sites in the rat heart (average signal of two independent DNase-Seq datasets). B Overlap between rat and mouse DNase-Seq peaks in the heart. Rat heart DNase-Seq peaks were converted to mouse $10 \mathrm{~mm}$ genome with LiftOver and then overlapped with mouse heart DNase-Seq peaks (ENCODE project dataset). $76 \%$ of the lifted-over rat DHS overlapped with the mouse ENCODE dataset. C IGV browser visualization of rat and mouse DNase-Seq reads at two syntenic genomic regions to illustrate the conservation of DNAse-Seq peaks between the two species. D Motif analysis at DNase I hypersensitive sites located within genes being downregulated or upregulated in WRF rats. Motifs with a $q$ value $<0.05$ are colored from yellow to red with red being the most significant. Lack of enrichment for a motif $(p>0.05)$ is displayed in gray. Diameter of each circle is also proportional to the enrichment $q$ value, with higher diameter for lower $q$ values. Transcription factors expressed in the rat heart are illustrated with a checkmark and those that are not expressed in the rat heart are labeled ne. $\mathbf{E}$ Expression profiles of several transcription factors whose motif is enriched in DNase-Seq peaks located in genes upregulated in WRF rats. Each dot represents individual fpkm values. Groups with different letters are significantly different ( $p<0.05$, Kruskal-Wallis test) 
Table 1 Transcription factors whose motif are enriched in genes up-regulated in WRF and their involvement in cardiovascular diseases

\begin{tabular}{|c|c|c|}
\hline Transcription factors & Role in cardiovascular diseases & References \\
\hline \multicolumn{3}{|l|}{ ETS family } \\
\hline ELK1, ELK4 & Involved in the cardiac gene regulatory network & [38] \\
\hline ETS1 & Mediates angiotensin II-related cardiac fibrosis and cardiac hypertrophy & {$[32,33]$} \\
\hline \multirow[t]{2}{*}{ ETV1 } & Induces atrial remodeling and arrhythmia & [34] \\
\hline & Determine fast conduction physiology in the heart & [39] \\
\hline ERG & Regulates endothelial-specific gene expression program & [40] \\
\hline \multicolumn{3}{|l|}{ NRF family } \\
\hline NRF1 & Loss in cardiomyocytes leads to apoptosis and reduced left ventricular function & [41] \\
\hline \multicolumn{3}{|l|}{ SP/KLF family } \\
\hline KLF3 & Involved in cardiovascular development and function & [35] \\
\hline KLF6 & Decreased expression in cardiomyocytes reduces cardiac fibrosis & [36] \\
\hline \multicolumn{3}{|l|}{ IRF family } \\
\hline IRF8 & Suppresses pathological cardiac remodeling & {$[37]$} \\
\hline
\end{tabular}

specific stages of disease progression (Fig. 5A, Additional file 2: Fig. S6) [25].

Restricting our analysis to modules of 50 genes or more (modules M0 to M37), we found that genes upregulated in the heart of WRF rats were enriched in five modules when compared to module M0, which comprised genes whose expression was unaffected by pressure overload (Fig. 5A, Additional file 1: Table S9). Several of these modules (M1, M11, and M16) include genes encoding proteins involved in ribosomes, translation, oxidative phosphorylation, and generation of precursor metabolites and energy (Fig. S6, Additional file 1: Tables S10, S11) [25]. Interestingly, these modules were preferentially activated from 3 days to 2 weeks after transverse aorta constriction, i.e., early after pressure overload rather than at advanced hypertrophy and heart failure stages [25].

To extend our analysis, we examined changes in gene expression in WRF rat hearts for each module. While gene expression within most modules was unaffected and similar to the control M0 module, we found that genes within 11 modules were globally either upregulated (8 modules) or downregulated (3 modules) (Fig. 5B, Additional file 1: Table S12). Most of these modules were identical to those identified with the enrichment analysis, confirming that lack of food intake during shift work in rat reprograms cardiac gene expression to transcriptional signatures associated with pressure overload. Interestingly, the same analysis performed using changes in gene expression in shift worker rats (W group) revealed that gene expression within eight modules was also misregulated when compared to other modules (Fig. 5C, Additional file 1: Table S13). Six out of eight modules (M1, M4, M7, M11, M16, and M27) were also upregulated in WRF rat heart, suggesting that shift work alone initiates changes in gene expression after 5 weeks and that these effects are exacerbated if animals do not eat food during work.

We also examined transcriptional signatures associated with ischemic cardiomyopathy (ICM) and dilated cardiomyopathy (DCM) in humans, using public datasets comparing gene expression profiles between post-mortem pathological heart samples with non-failing heart samples [42]. We found that genes upregulated in ICM hearts were globally upregulated in WRF rat heart, while those downregulated by ICM were slightly downregulated in WRF rat heart (Fig. 5D). The effects were less pronounced for genes being misregulated by DCM (Additional file 2: Fig. S7), and no difference in gene expression profile was observed for shift worker rats (Fig. 5D). Taken together, these data indicate that lack of food intake during shift work induces changes in cardiac gene expression that resemble transcriptional signatures observed in pathological heart samples.

Closer inspection of the genes whose expression is altered in WRF rat heart revealed that many of them are associated with cardiovascular disorders. These include genes whose products are components of the extra-cellular matrix (ECM) such as collagen type 1 alpha 1 chain (Col1a1) and collagen type 1 alpha 2 chain (Col1a2), which are all upregulated in WRF rat heart (Fig. 5E). Because fibroblast markers including Thy-1 cell surface antigen (Thy1; Fig. 5E) and Fibroblast-specific protein-1 (S100a4; Fig. 5F) are also significantly upregulated in WRF rat heart, this suggests that lack of food intake during shift work may lead to collagen deposition and cardiac fibrosis. S100 proteins are calcium-binding proteins that regulate macrophage inflammation and whose expression is increased in the injured myocardium $[43,44]$. Given that three other members of the S100 family (Fig. 5F), and that several chemokines are also 


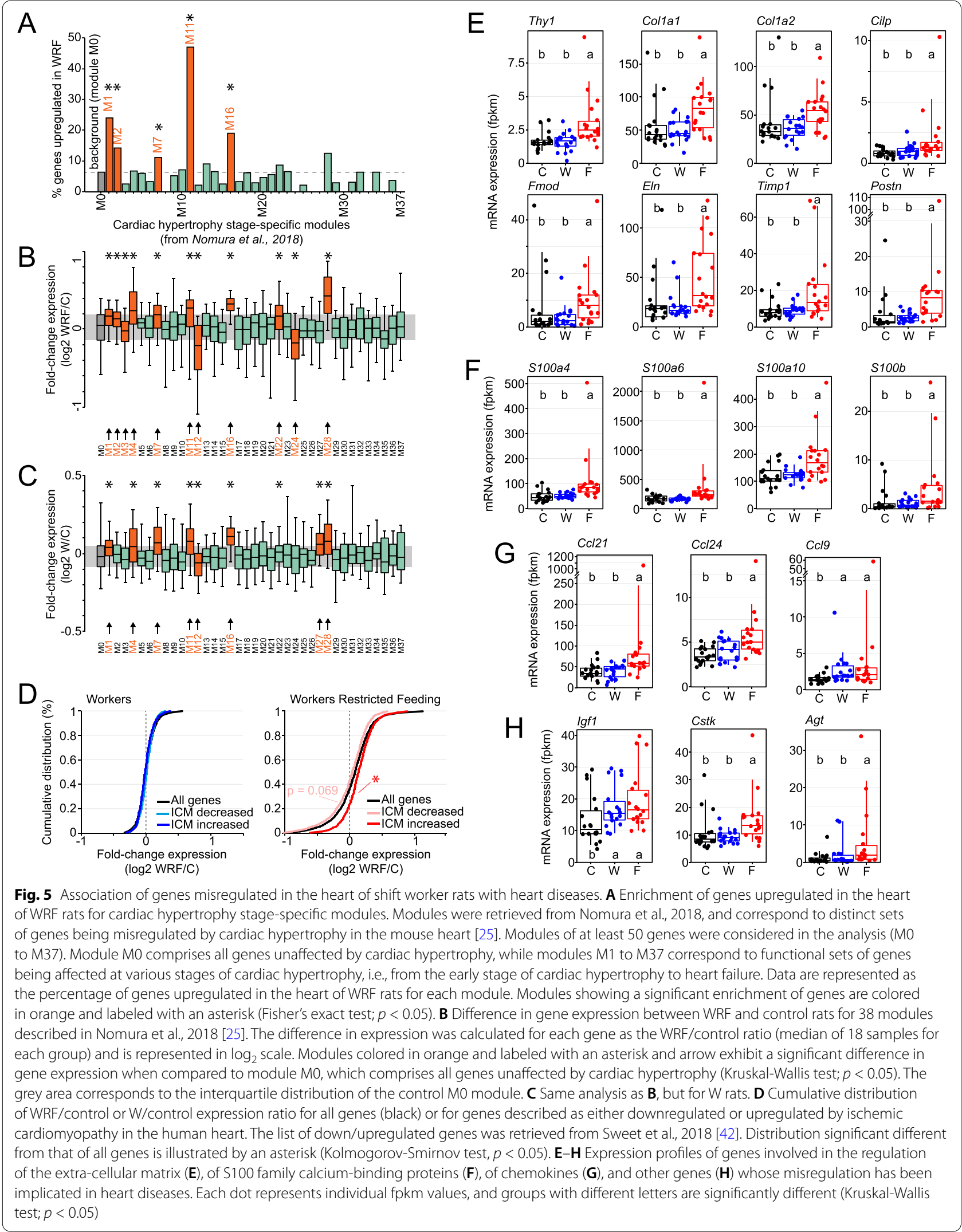


upregulated in WRF rats (Fig. 5G), this suggests that the heart of WRF rats is inflamed and colonized by immune cells. Finally, several genes whose increased expression has been shown to lead to cardiac hypertrophy and heart diseases are also upregulated in WRF rat heart, including Insulin-like growth factor 1 (Igf1), Angiotensin (Agt), and Cathepsin K (Ctsk) (Fig. 5H) [45-48]. Interestingly, the hormone Igfl and the inflammatory chemokine $C c l 9$ are also upregulated in the heart of W rats, suggesting that shift work alone may also initiate some cardiac injury and inflammation in the heart (Fig. 5G, H).

\section{Shift work induces collagen deposition in the heart}

Based on our findings that genes whose expression is altered by shift work are also misregulated in pathological heart samples from mice and humans, and since many of them regulate immune responses and ECM composition, we next examined if hearts from W and WRF rats displayed signs of inflammation and cardiac fibrosis.

First, we performed immunohistochemistry of two immune cell markers, CD45 and CD68, in the heart of W, WRF, and C rats. CD45 detects most cells of hematopoietic origin at all stages of maturation, while CD68 is a marker of inflammation associated with the involvement of monocytes/macrophages $[49,50]$. Consistent with the transcriptional response observed in WRF but not W rat hearts, the number of both CD45- and CD68-positive cells was significantly higher in the heart of WRF rats than that of $\mathrm{W}$ and control rats (Fig. 6A, B).

Next, we performed immunohistochemistry of Collagen 1 to determine whether shift work leads to cardiac fibrosis. Surprisingly, the hearts of both W and WRF rats showed increased Collagen 1 expression (Fig. 6C), i.e., W rat heart exhibited signs of fibrosis despite any increase in Col1a1 and Col1a2 expression (Fig. 5E). To confirm this result, we conducted picrosirius red staining of all $C, W$, WRF rat hearts ( $n=18$ per group), and doubleblind scored collagen deposition based on a 0 to 4 scale (0: no collagen deposition; 4: maximum collagen deposition; Additional file 2: Fig. S8). Analysis of picrosirius red staining confirmed Collagen 1 immunohistochemistry, i.e., collagen deposition was significantly higher in $\mathrm{W}$ and WRF hearts compared to $\mathrm{C}$ rat hearts (Fig. 6D).

Taken together, these results indicate that while lack of food intake during shift work (but not shift work alone) leads to increased cardiac inflammation, 5 weeks of shift work alone is sufficient to promote cardiac fibrosis. They also suggest that shift work induces some heart damages

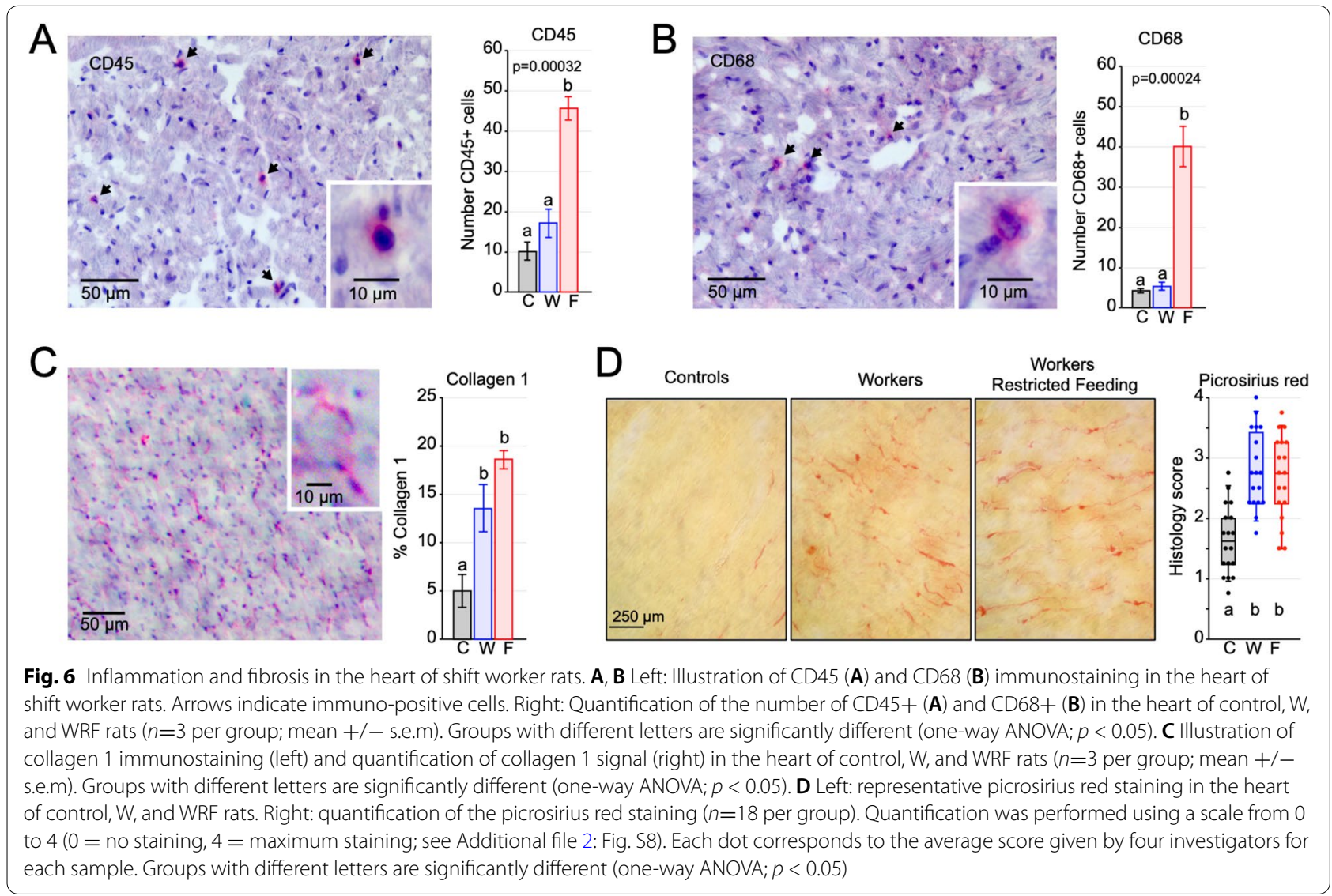


and that these effects are potentiated if animals do not have access to food during work.

\section{Discussion}

Several epidemiological studies have shown that shift workers have an increased risk of developing cardiovascular diseases [11, 13-15]. However, the underlying mechanisms are unknown as no study has directly investigated in an animal model how shift work affects heart biology. Using a well-characterized model of shift work in rats [22], we provide evidence that shift work reprograms the heart cycling transcriptome and leads to cardiac fibrosis. Importantly, our data also demonstrate that lack of food intake during shift work, an intervention that prevents obesity and diabetes in rat shift workers, does not preclude cardiac fibrosis. Instead, restricting food access during shift work markedly disrupts gene expression in the heart and leads to cardiac fibrosis and inflammation. Together, our findings provide insights into how shift work affects cardiac functions and strongly suggest that strategies aiming at mitigating the development of metabolic disorders in shift workers may have adverse effects on cardiovascular diseases.

Our genome-wide analysis of gene expression revealed that shift work reprograms the heart cycling transcriptome. In shift worker rats, the number of rhythmic genes is decreased by half and the molecular clock oscillation is altered. Intriguingly, this alteration of clock gene rhythmic expression originates primarily from differences between the three shift work protocol replicates rather than a global dampening of clock oscillations in every rat (Additional file 2: Fig. S2B). As shown previously, shift work in rats shifts the hepatic clock rhythms due to the reversal of the food intake rhythm, i.e., because shift workers eat preferentially during their work shift $[20,22]$. Given that peripheral clocks can be synchronized by multiple cues including rhythms in food intake, hormones, body temperature, and neuronal activity, the heart clock may be less sensitive to changes in rhythmic food intake than the liver clock, and more sensitive to other cues such as rhythms in locomotor activity or in neuronal activity that originate from the SCN [22]. This possibility is supported by findings showing that PER1 rhythmic expression is shifted by shift work in some hypothalamic structures (e.g., arcuate nucleus, dorsomedial hypothalamus), but not in others (e.g., SCN, paraventricular nucleus) [51]. However, why replicated rhythms did not respond homogenously still remain unclear and warrant further investigation in future experiments. Given the recent demonstration that liver-clock disruption (e.g., because of daytime feeding) modulates the transcriptional rhythmicity of peripheral tissues [52], it is also possible the shift work-induced reversal of liver clock oscillation decreases the rhythmicity of the heart transcriptome in shift worker rats.

The diversity in cues that can synchronize peripheral clocks may also explain why most clock genes exhibit dampened rhythmic expression in WRF rat hearts (Fig. 2D, Additional file 2: Fig. S2A). The synchronizing effect of rhythmic food intake may indeed be counteracted by antiphasic cues driven by the sleep/wake cycle, thereby reducing the amplitude of the circadian clock oscillation [53]. Since time of scheduled exercise and muscle contraction modulate the expression of clock genes in the skeletal muscle independently of the SCN $[54,55]$, locomotor activity during the rest phase alone may also contribute to the observed dampened clock gene oscillation in WRF rat hearts. Interestingly, although the molecular clock oscillations are dampened in WRF rat hearts, the number of rhythmic genes is twofold higher than in control rat hearts. Because rhythmic gene expression can be driven by feeding rhythms independently of the molecular clock [56-58], this increased rhythmic gene expression in the heart of WRF rats could be caused by the $8 \mathrm{~h}$ of fasting occurring during work. This would explain why rhythms in gene expression occur at discrete phases rather than across the 24-h day. Consistent with this possibility, the expression of many genes involved in oxidative phosphorylation is decreased at the end of shift work, likely reflecting a decrease in substrates for the generation of ATP. Moreover, genes involved in endocytosis are increased during shift work, maybe as a result of compensatory mechanisms aimed at increasing the uptake of extracellular molecules for energy sources [59]. Another factor that may contribute to the higher number of rhythmically expressed genes in WRF rat heart is the increased immune cell infiltration in their heart (Fig. 6A, B), i.e, the increased heterogeneity of cardiac cells may account for the additional number of rhythmically expressed genes. Diurnal rhythms in immune cells trafficking have been described, with for example higher number of Ly6 $\mathrm{C}^{\mathrm{hi}}$ inflammatory monocytes around ZT8 in mouse blood [60]. This could also explain why genes rhythmic in the heart of WRF rats are enriched in TGF-beta and Toll-like receptor signaling pathways (Fig. 2E).

In addition to dampening the molecular clock oscillations and reprogramming the cycling heart transcriptome, lack of food intake during shift work strongly impairs gene expression in the heart. Because these effects are less pronounced in shift worker rats, impaired gene expression in WRF rat hearts is likely due to the conflicting information between the work schedule, where animals are awake and likely to have increased heart workload due to increased sympathetic neural activity [61], and the lack of food intake. Motif 
analysis in DNase-Seq peaks located in misregulated genes in WRF rats revealed that transcription factors of the ETS family are likely involved (Fig. 4D). ETS transcription factors are key regulators of cell proliferation, differentiation, angiogenesis, and inflammation, and their transcriptional activity is regulated by signaling pathways including the MAP kinase pathway [62, 63]. Interestingly, our KEGG analysis pathway revealed that components of the MAPK signaling pathway are rhythmically expressed in $C$ rats but not in WRF rats and that they are also downregulated in the heart of WRF rats (Figs. 2E and 3D). Importantly, ETS transcription factors contributes to many aspects of the heart physiology, and alteration of their transcriptional activity is associated with cardiovascular diseases including cardiac fibrosis and arrhythmia [32-34, 38-40]. Other transcription factor motifs were identified, including SP/KLF family members and IRF8. Interestingly, these transcription factors are involved in the regulation of heart physiology and some of them were shown to promote cardiac diseases when their activity is impaired (Table 1).

Several biological pathways that are upregulated in WRF rat hearts are involved in protein synthesis and degradation, DNA synthesis, and gene expression (e.g., purine and pyrimidine metabolism). Interestingly, these processes are increased in the early stages of cardiac hypertrophy, suggesting that 5 weeks of shift work without food intake during work impairs heart functions $[25,27,64]$. Consistent with this possibility, many genes misregulated in WRF rat heart are also misregulated in cardiomyocytes several days after transverse aorta constriction, i.e., when the heart is under pressure overload and in the early stage of cardiac hypertrophy, and before its progression to heart failure [25]. Moreover, many genes upregulated in WRF rat hearts are known to be misregulated in ailing hearts (Fig. 5E-H). For example, increased expression of Col1a1 and Col1a2, which encode for components of type I collagen, has been linked to remodeling of the cardiac matrix [65], and upregulation of collagen 1 is a well-established marker of cardiac fibrosis and is observed after cardiac hypertrophy [66-68]. This upregulation of Col1a1 and Colla2 expression, along with other markers of cardiac fibrosis like Thy1, Postn, and Cilp [69-71], is consistent with our findings that the heart of WRF rat is fibrotic (Fig. 6C, D). In addition, the increased expression of several chemokines and members of the $\mathrm{S} 100$ protein family, along with the increased number of $\mathrm{CD} 45+$ and CD68+ cells, also indicate that the heart of WRF rats is inflamed and colonized by immune cells. Given that inflammation of the myocardium also occurs after hypertrophic cardiopathy [72, 73], our data altogether strongly suggest that lack of food intake during shift work is associated with cardiac hypertrophy.

Time-restricted feeding is recognized as an intervention that can prevent the development of metabolic diseases against diverse nutritional challenges [74-77]. In that regard, restricting food consumption to the night in a model of shift work in rats prevents shift work-induced increased fat content and impaired glucose tolerance [20, 21]. While our data suggest that time-restricted feeding potentiates the adverse effects of shift work on the heart transcriptome and cardiac inflammation, it is possible that the duration for which rats did not have access to food (i.e., $8 \mathrm{~h}$ of shift work) was insufficient to achieve the health benefit of time-restricted feeding. Future experiments aiming at testing the duration of time-restricted feeding during shift work on the heart biology will be needed to test this possibility.

While our initial RNA-Seq analysis did not identify large defects in gene expression in the heart of $\mathrm{W}$ rats, our analysis of modules associated with pressure overload, cardiac hypertrophy, and its progression to heart failure uncovered significant differences between shift worker and control rats [25]. This discrepancy is likely due to our stringent analysis of differential gene expression, which comprised a Benjamini-Hochberg $p$ value correction because of the multiple comparisons (Additional file 2: Fig. S4). Most modules exhibiting impaired gene expression in W rats were also found in WRF rats, suggesting that shift worker rats were in the early stage of developing cardiac diseases. Consistent with this possibility, hearts of shift worker rats displayed cardiac fibrosis, but no sign of inflammation. While it still remains unclear why W rat hearts exhibit increased collagen deposition without changes in Col1a1 and Col1a2 mRNA expression, their expression may be acutely upregulated by shift work, i.e., their expression is similar to those observed in $\mathrm{C}$ rats during weekends when the tissues were collected. Future experiments will be required to test this possibility.

\section{Conclusions}

In summary, our results indicate that 5 weeks of shift work in rats are sufficient to promote cardiac fibrosis, while the effects of shift work are exacerbated when animals do not have access to food during work. Based on these data, it is tempting to speculate that the differences we observed between W and WRF shift worker rats may be relevant to explain some of the heterogeneity that has been reported in epidemiological studies of human shift workers [10, 12]. Importantly, our findings also suggest that lack of food intake during work, which was proposed as a possible intervention to prevent the development of metabolic disorders in shift workers, may not be 
appropriate to prevent the development of cardiovascular diseases in shift workers.

\section{Methods \\ Animals}

Seven-to-eight-week-old male Wistar rats were exposed to a shift work protocol for 5 weeks. Each animal was housed individually in a $12 \mathrm{~L}: 12 \mathrm{D}$ cycle $(7$ am to $7 \mathrm{pm})$ throughout the protocol (Fig. 1). The protocol consisted of 54 animals randomly assigned to three groups: 18 Controls ' $\mathrm{C}$ ' (undisturbed and no shift work protocol, ad libitum food and water), 18 Workers 'W' (shift work protocol, ad libitum food and water), 18 Worker Restricted Feeding 'WRF' (shift work protocol, ad libitum water, access to food only outside shift work). Shift work protocol consisted of five days of scheduled 'work' (workday: Monday to Friday, 9 am to $5 \mathrm{pm}$ ) followed by 2 days without 'work' and given ad libitum food and water (weekend: Saturday and Sunday).

\section{Shift work protocol}

To simulate shiftwork, rats were placed in a slowly rotating drum that was designed to keep the animal awake during their natural rest phase, as previously described [22]. Briefly, drums rotated with a speed of one revolution per $3 \mathrm{~min}$ and enabled animals to move, eat, and drink freely. However, animals were not able to fall asleep and had to stay awake without requiring them to exhibit laborious movements. Food and water were hung from a concentric middle tube and freely available, unless noted otherwise for the WRF group. Outside of work hours, rats were housed in their individual cages with food and water provided ad libitum and kept under 12:12 LD cycle.

\section{Heart tissue collection}

After 5 weeks of shift work protocol, rats were euthanized on a Saturday every $4 \mathrm{~h}$ starting $1 \mathrm{~h}$ after light on (ZT01) at $7 \mathrm{am}$. Hearts were collected, directly flash-frozen in liquid nitrogen, and shipped to Texas A\&M University.

\section{Whole transcriptome sequencing (RNA-Seq)}

Frozen hearts (atria and ventricles) were crushed to smaller pieces in liquid nitrogen, and RNA was isolated using Trizol reagent according to the manufacturer's instructions (Life Technologies). PolyA mRNA were then purified from $4 \mu \mathrm{g}$ of total RNA using Dyna oligo dT beads following manufacturer's instructions (Invitrogen). Sequencing libraries were constructed using NEB Ultra Directional RNA Library Prep Kit from Illumina (New England BioLabs) following manufacturer's instructions. Briefly, polyA purified RNA was fragmented for $15 \mathrm{~min}$ at $94^{\circ} \mathrm{C}$ before first- and second-strand cDNA synthesis. Purification of cDNA was done using AMPure XP beads following NEB Ultra Directional RNA Library Prep Kit instructions. Libraries were then PCR-amplified for 13 cycles, bead-purified, and quantified using a Quantus Fluorometer (Promega) and qPCR. Libraries were generated with multiple bar-coded adaptors, mixed in equimolar ratio, and sequenced on three lanes of an Illumina NextSeq 500 with a sequencing length of $76 \mathrm{bps}$.

\section{Rat heart DNase-Seq}

Nuclei were isolated from crushed frozen heart samples of control $C$ rats ( $n=2$ biological replicates) using a dounce homogenizer in lysis buffer $(0.32 \mathrm{M}$ sucrose, $10 \mathrm{mM}$ Tris- $\mathrm{HCl} \mathrm{pH} \mathrm{8,5} \mathrm{mM} \mathrm{CaCl} 2,5 \mathrm{mM} \mathrm{MgCl} 2,2$ mM EDTA, 0.5 mM EGTA, 1 mM DTT). Samples were homogenized 6-times with pestle $\mathrm{A}$ and 4-times with pestle B. Homogenates were mixed with sucrose lysis buffer $(2.2 \mathrm{M}$ sucrose, $10 \mathrm{mM}$ tris $\mathrm{HCl} \mathrm{pH} 8,5 \mathrm{mM}$ $\mathrm{CaCl} 2,5 \mathrm{mM} \mathrm{MgCl} 2,2 \mathrm{mM}$ EDTA, $0.5 \mathrm{mM}$ EGTA, 1 $\mathrm{mM}$ DTT) and then layered on top of a sucrose cushion (2.05 M sucrose, 10\% glycerol, $10 \mathrm{mM}$ HEPES pH7.6, $15 \mathrm{mM} \mathrm{KCl}, 2 \mathrm{mM}$ EDTA, 1mM PMSF, $0.15 \mathrm{mM}$ spermine, $0.5 \mathrm{mM}$ spermidine, $0.5 \mathrm{mM}$ DTT). Samples were ultracentrifuged for $45 \mathrm{~min}$ at 24,000 rpm (100,000 g) at $2^{\circ} \mathrm{C}$ in a Beckmann SW28 rotor. The supernatant was removed and nuclei were washed three-times in resuspension buffer (10 mM Tris, $\mathrm{pH} 7.5,150 \mathrm{mM} \mathrm{NaCl}, 2$ mM EDTA, $1 \mathrm{mM}$ PMSF). Nuclei were then counted, aliquoted (five million nuclei per tube), and flash-frozen. On the day of DNase digestion, nuclei were thawed, pelleted, and digested for $3 \mathrm{~min}$ at $37^{\circ} \mathrm{C}$ in DNase I digestion buffer $(6 \mathrm{mM} \mathrm{CaCl} 2,78.5 \mathrm{mM} \mathrm{NaCl}, 13.5 \mathrm{mM}$ Tris- $\mathrm{HCl} \mathrm{pH} 8,54 \mathrm{mM} \mathrm{KCl}, 0.9 \mathrm{mM}$ EDTA, $0.45 \mathrm{mM}$ EGTA, 0.45 spermidine) supplemented with $4 \mu \mathrm{l}$ of DNase I ( 80 units $/ \mathrm{mL}$ ). After digestion, an equal volume of stop buffer $(50 \mathrm{mM}$ Tris- $\mathrm{HCl} \mathrm{pH} 8,100 \mathrm{mM} \mathrm{NaCl}$, $0.1 \%$ SDS, $100 \mathrm{mM}$ EDTA, $1 \mathrm{mM}$ spermidine, $0.3 \mathrm{mM}$ spermine) was added to each tube and incubated at $55^{\circ} \mathrm{C}$ for $1 \mathrm{~h}$. RNA was digested by adding $4.5 \mu \mathrm{l}$ of RNase A $(10 \mathrm{mg} / \mathrm{mL})$ and samples were incubated at $37^{\circ} \mathrm{C}$ for $30 \mathrm{~min}$. Samples were purified by phenol/chloroform extraction and resuspended in water. DNA size fractionation was done using a sucrose gradient and centrifuged for $30,000 \mathrm{rpm}$ for $20 \mathrm{~h}$ at $20^{\circ} \mathrm{C}$ in an SW-41 rotor with no brake and minimum acceleration. DNA fractions with fragment size under $500 \mathrm{bp}$ were pooled together, ethanol purified, and resuspended in water. DNase-Seq libraries were generated using NEBNext ${ }^{\circledR}$ ChIP-Seq Library Prep Master Mix Set NEB) as per the manufacturer's instructions. DNA collected from the DNase I digestion was quantified using a Quantus Fluorometer (Promega), and $10 \mathrm{ng}$ was used to generate the libraries. Libraries were PCR-amplified for 14 cycles using Phusion Taq (M0530S). Adaptor dimers were removed using 
gel purification, and libraries were purified by phenolchloroform extraction followed by ethanol precipitation. Libraries were quantified using a Quantus fluorometer and $\mathrm{qPCR}$ and subjected to paired-end sequencing $(2 \mathrm{x}$ 75bp) using an Illumina NextSeq 500.

\section{Alignment of RNA-Seq libraries and differential gene expression analysis}

Libraries were sequenced to a median depth of $\sim 25$ million reads/sample and mapped to the Rattus norvegicus Rn6 genome assembly using Tophat 2 alignment and the following criteria: --read-realign-edit-dist 2 -g 1 --b2-sensitive [78]. Sequencing depth and percentage alignment are provided in Additional file 1: Table S14. Gene expression was analyzed using Cufflinks with the following criteria: --library-type fr-firststrand [79]. Visualization of the forward and reverse strands were done using a custom shell script with total signal normalized to 10 million reads and viewed with the Integrated Genome Viewer [80]. Only genes expressed above a fpkm threshold of 1 in at least 18 samples out of the 54 total samples were considered for statistical analysis ( $n=10,257$ genes). For the differential gene expression analysis, fpkm values of all 18 animals for each group were combined (without considering the time of tissue collection) and then compared between groups using a Kruskal-Wallis test with Benjamini-Hochberg correction for multiple comparisons followed by a Mann-Withney $U$ test post hoc analysis to identify differences between groups. Genes were considered to be expressed differently between group if $q \leq 0.05$.

\section{Alignment of DNase-Seq libraries and motif analysis}

Libraries were sequenced to a median depth of $\sim 36$ million and mapped to the Rattus norvegicus Rn6 genome with bowtie2 using the following criteria: $-\mathrm{t}-$ phred33 -X 650 [81]. The average alignment was of $\sim 85 \%$. Sam files were converted to bam files and merged using SAMtools view, sort, and merge [82]. Duplicates were removed with Picard 2.8.2 MarkDuplicatesWithMateCigar created by the Broad Institute. A custom python script was used to remove unpaired reads, unmapped reads, and filled in sequences between paired-end reads. Sorted coordinates were then made into BWfiles using Bedtools, normalized to 10 million reads, and viewed with the Integrated Genome Viewer [80, 83]. Peak calling was done using findPeaks from the HOMER suite using the following commands: -size 45 -ntagThreshold 2 -region [31]. Open chromatin regions for genes of interest were determined by using HOMER gene annotation and annotation for these genes were extended $10 \mathrm{~kb}$ from transcription start sites and $1 \mathrm{~kb}$ from transcription termination sites. These gene windows were overlapped with DNase-Seq reads using intersectBed from Bedtools suite [83]. Motif analysis was done on these overlapping regions using the perl script findMotifsGenome.pl from HOMER using the following command: -size given [31]. Motif analysis in open chromatin regions located in rhythmically expressed genes was performed similarly and using the list of 28,514 DNase I hypersensitive sites in rat heart as background (Additional file 1: Table S6). Mouse heart DNase-Seq datasets were downloaded from the ENCODE project website (https://www.encodeproject.org/experiments/ ENCSR000CNE/) and analyzed as above using mm10 as reference genome [28].

\section{Analysis of rhythmic gene expression}

Rhythmicity analysis was performed using six algorithms from four programs: F24 [84, 85], JTK_CYCLE, ARSER, and LS from MetaCycle [86], HarmonicRegression [87], and RAIN [88]. The resulting $p$ values from all 6 algorithms were combined using Fisher's method into one $p$ value, which was then adjusted to control for the falsediscovery rate (FDR) using the Benjamini-Hochberg method [89] within the p.adjust function available in base R. Genes with a $q$ value under 0.05 were considered as rhythmically expressed. We also performed a differential analysis of rhythmicity using dryR, using standard parameters [23].

\section{KEGG pathway and Gene Ontology analyses}

All KEGG and GO analyses were performed using the kegga and goana functions available within the $\mathrm{R}$ package Limma [90]. Gene symbols were converted to Entrez IDs with AnnotationDbi [91].

\section{Effects of shift work on cardiac hypertrophy stage-specific modules}

The identity of the genes clustered into the 56 cardiac hypertrophy stage-specific modules was retrieved from Nomura et al., 2018 (Supplementary Data 4 [25];), and cross-referenced with our list of 10,257 genes expressed in the rat heart. To avoid statistical bias due to low number of genes in a module, we restricted our analysis to modules of 50 genes or more (modules M0 to M37). Enrichment was calculated as the percentage of genes being upregulated in the heart of WRF rats for each module and considered significant if $p \leq 0.05$ when analyzed with a Fisher's exact test. Differences in gene expression between WRF and C rats (Fig. 5B) and between W and C rats (Fig. 5C) were calculated for each gene as the WRF/ control ratio (median of 18 samples for each group). Differences in gene expression between the control module M0 and the modules M1 to M37 were analyzed using a Kruskal-Wallis test and considered significant if $p \leq 0.05$. 


\section{Effects of shift work on the expression of genes being misregulated by ischemic cardiomyopathy and dilated cardiomyopathy}

The list of genes being down/upregulated in the heart of patients suffering from ischemic cardiomyopathy (ICM) and dilated cardiomyopathy (DCM) was retrieved from Sweet et al., 2018 (Table S3 [42];). This list was then cross-referenced with our list of 10,257 genes expressed in the rat heart. Differences in the distribution between all genes and genes either up/downregulated by ICN and DCM were assessed using Kolmogorov-Smirnov test and considered significant if $p<0.05$. Distributions were normalized to 500 quantiles to reduce the effect of size on the Kolmogorov-Smirnov test sensitivity [92].

\section{Immunohistochemistry}

Immunohistochemistry was performed as previously described with some minor modifications [93, 94]. Serial $10-\mu \mathrm{m}$ thick cryosections of the heart $(n=3$ per group; C group rats: C29ZT17, C33ZT1, C36ZT13; W group rats: W5ZT9, W17ZT1, W36ZT13; WRF group rats: WRF42ZT13, WRF46ZT1, WRF51ZT9) were airdried overnight and fixated in fresh acetone for $15 \mathrm{~min}$ at room temperature. Slides were then air-dried for 10 min and hydrated for $5 \mathrm{~min}$ in water and $5 \mathrm{~min}$ in PBS. Nonspecific binding was then blocked by incubation with PBS containing 2\% BSA (fraction V, globulin free; SigmaAldrich) for $30 \mathrm{~min}$. Endogenous biotin was blocked by the addition of streptavidin and biotin solutions, following the manufactures' instructions (Streptavidin/ Biotin Blocking Kit, Vector Laboratories, Burlingame, CA). Slides were then incubated with primary antibodies, anti-CD45 (5 $\mu \mathrm{g} / \mathrm{mL}$, MCA340R, OX-33, mouse IgG1, Bio-Rad), anti-CD68 (5 $\mu \mathrm{g} / \mathrm{mL}, \mathrm{MCA341R,} \mathrm{ED1,}$ mouse IgG1, Bio-Rad), or anti-Collagen $1 \alpha 1$ (1 $\mu \mathrm{g} /$ $\mathrm{mL}, \mathrm{NB} 600-408$, rabbit IgG, Novus Biologicals) in 0.5\% $\mathrm{NP} 40$ and $0.05 \%$ SDS overnight at $4^{\circ} \mathrm{C}$. Isotype-matched irrelevant mouse monoclonal antibodies or irrelevant polyclonal antibodies (BD Biosciences or Jackson ImmunoResearch Laboratories) were used as controls. Slides were then quickly rinsed twice in PBS and then $3 \times 5$ min in PBS. Primary antibodies were then detected with biotin-labeled $\mathrm{F}(\mathrm{ab}$ ') 2 anti-mouse IgG (Jackson ImmunoResearch Laboratories) or anti-rabbit IgG (Southern Biotechnology Associates) in PBS containing 2\% BSA, as appropriate for $30 \mathrm{~min}$. Slides were quickly rinsed twice in PBS and then $3 \times 5$ min in PBS, before being incubated for 30 min with 1/500 ExtraAvidin-Alkaline Phosphatase (Sigma-Aldrich) in PBS containing 2\% BSA. Slides were then rinsed with PBS and incubated for 10 min with 200 $\mathrm{mM}$ TRIS pH 8.3. Staining was then developed with Vector Red Alkaline Phosphatase Kit (Vector Laboratories) for $10 \mathrm{~min}$. Sections were then counterstained for $1 \mathrm{~min}$ with Gill's hematoxylin No. 3 diluted $1 / 5$ with water (Sigman-Aldrich) and were then rinsed in water. Slides were dehydrated through $95 \%$ and $100 \%$ ethanol, cleared with xylene, and mounted with Permount (VWR).

The number of positive cells was assessed by two individuals counting 10 blinded selected fields per section with a random start position. Collagen was analyzed using Image J software using the standard algorithms to define the areas of staining (Rasband, W. S., ImageJ U.S. National Institute of Health, Bethesda, MD). Raw data are provided in Additional file 1: Table S14.

\section{Picrosirius red staining}

Characterization of fibrosis was performed using picrosirius red, which specifically binds to collagen, on all 54 heart samples as previously described [93, 94]. Heart samples were embedded in Tissue-Tek OCT, sliced $10-\mu \mathrm{m}$ thick with a cryostat and slices were air-dried to slides. Slides were stained in picrosirius red for $1 \mathrm{~h}$, washed in acidified water twice, and dehydrated in three changes of $100 \%$ ethanol. Slides were then cleared with xylene and mounted with a resinous medium.

Quantification was performed using a scale from 0 to $4(0=$ no staining, $4=$ maximum staining $)$ as shown in Additional file 2: Fig. S8, by four investigators doubleblinded to the conditions. The final score for each animal corresponds to the average score given by all four investigators. Raw data are provided in Additional file 1: Table S15.

\section{Supplementary Information}

The online version contains supplementary material available at https://doi. org/10.1186/s12915-022-01256-9.

Additional file 1: Table S1. Fpkm data, analysis of rhythmicity and gene expression levels (related to Figs. 2, 3, and S3). Table S2. dryR analaysis of differential rhythmicity in gene expression (related to Fig. 2). Table S3. KEGG pathway analysis, rhythmically expressed genes (related to Fig. 2). Table S4. List of genes being rhythmically expressed in each enriched KEGG pathway (related to Fig. 2 and S2). Table S5. KEGG pathway analysis, differentially expressed genes (related to Fig. 3). Table S6. List of DNase I hypersensitive sites in rat heart and genomic location (related to Fig. 4). Table S7. Motif analysis for genes being up-regulated or down-regulated in WRF rats (related to Fig. 4). Table S8. Motif analysis for genes being rhythmically expressed in C, W, and WRF rat hearts (related to Fig. S5). Table S9. Results statistical analysis for Fig. 5A (related to Fig. 5). Table S10. KEGG pathway analysis of the genes assigned to the modules M0 to M37 (related to Fig. 5). Table S12. Results statistical analysis for Fig. 5B (related to Fig. 5). Table S13. Results statistical analysis for Fig. 5B (related to Fig. 5). Table S14. Quantification of picrosirius red staining (related to Fig. 6). Table S15. Quantification of CD45, CD68, and Collagen 1 staining (related to Fig. 6). Table $\mathbf{S 1 6}$. Summary of RNA-Seq library size and percentage alignment (related to Methods).

Additional file 2: Figure S1. Effect of shift work on the cycling heart transcriptome using a differential analysis of rhythmicity with dryR (related to Fig. 2). Figure S2. Effect of shift work on the expression of core clock genes in the rat heart (related to Fig. 2). Figure S3. Effect of shift work on 
the rhythmic expression of genes accounting for enriched KEGG pathways (related to Fig. 2). Figure S4. Analysis of differential gene expression between C, W, and WRF rats (related to Fig. 3). Figure S5. Motif analysis at DNase I hypersensitive sites located within genes rhythmically expressed in the heart of C, W, and WRF rats (related to Fig. 4). Figure S6. KEGG pathway analysis on modules M0 to M37 (related to Fig. 5). Figure S7. Effect of shift work on the expression of genes misregulated by dilated cardiomyopathy (related to Fig. 5). Figure S8. Quantification of picrosirius red staining in the heart (related to Fig. 6).

Additional file 3: Table S11. Gene Ontology analysis of the genes assigned to the modules M0 to M37 (related to Fig. 5).

\section{Acknowledgements}

We are grateful to Cynthia Córdoba-Manilla for her assistance with the shift work protocol and tissue collection. We are thankful to Michael Rosbash, Kate Abruzzi, and Ryanne Spann for helping with the sequencing of the RNA-Seq libraries. We also thank the Texas A\&M Institute for Genome Sciences and Society for helping with sequencing the DNase-Seq libraries and for the maintenance of their high-performance computing cluster. We are grateful to Matt Sachs for helping with setting up the DNase-Seq technique and to Darrell Pilling for helping with immunohistochemistry.

\section{Authors' contributions}

A.J.T., R.M.B., and J.S.M. conceived and designed the experiments. N.N.G.V., C.E., and R.M.B. performed the shift work experiment and collected the heart samples. A.J.T. generated the RNA-Seq and DNase-Seq libraries and performed the picrosirius red staining. A.J.T. and T.R.K. performed the immunohistochemical assays. A.J.T., B.J.G., and J.S.M. performed the bioinformatics analysis. A.J.T. and J.S.M. wrote the manuscript, and all authors contributed to editing the final draft. The authors read and approved the final manuscript.

\section{Funding}

This work was supported by startup funds from Texas A\&M University, a Texas A\&M University-CONACYT Research Grant (2015-033), and in part by the National Institutes of Health (R21 Al144454 to J.S.M) and by a APIIT-IG201321 grant to E.C. and R.M.B.

\section{Availability of data and materials}

All data generated or analyzed during this study are included in Additional files. Sequencing datasets have been deposited on Gene Expression Omnibus (GSE124870) and can be accessed using the following link: https://www.ncbi. nIm.nih.gov/geo/query/acc.cgi?acc $=$ GSE124870

Public access is currently restricted. However, datasets can still be accessed using the token: evkzwoiortunlmv.

\section{Declarations}

\section{Ethics approval and consent to participate}

Experiments were approved by the Universidad Nacional Autonoma de Mexico, in accordance with animal handling, Norma Oficial Mexicana NOM-062-ZOO-1999.

\section{Consent for publication}

Not applicable.

\section{Competing interests}

The authors declare that they have no competing interests.

\section{Author details}

1'Department of Biology, Texas A\&M University, College Station, TX 77843, USA. ${ }^{2}$ Program of Genetics, Texas A\&M University, College Station, TX 77843, USA. ${ }^{3}$ Center for Biological Clock Research, Texas A\&M University, College Station, TX 77843, USA. ${ }^{4}$ Departamento de Anatomía, Facultad de Medicina, Universidad Nacional Autónoma de México, Ciudad Universitaria, Mexico City, Mexico. ${ }^{5}$ Instituto de Investigaciones Biomédicas, Universidad Nacional Autónoma de México, Ciudad Universitaria, Mexico City, Mexico.
Received: 18 June 2021 Accepted: 16 February 2022

Published online: 03 March 2022

\section{References}

1. Wang XS, Armstrong ME, Cairns BJ, Key TJ, Travis RC. Shift work and chronic disease: the epidemiological evidence. Occup Med (Lond). 2011;61(2):78-89.

2. Schernhammer ES, Kroenke CH, Laden F, Hankinson SE. Night work and risk of breast cancer. Epidemiology. 2006;17(1):108-11.

3. Schernhammer ES, Laden F, Speizer FE, Willett WC, Hunter DJ, Kawachi I, et al. Rotating night shifts and risk of breast cancer in women participating in the nurses' health study. J Natl Cancer Inst. 2001;93(20):1563-8.

4. Antunes LC, Levandovski R, Dantas G, Caumo W, Hidalgo MP. Obesity and shift work: chronobiological aspects. Nutr Res Rev. 2010;23(1):155-68.

5. Karlsson B, Alfredsson L, Knutsson A, Andersson E, Toren K. Total mortality and cause-specific mortality of Swedish shift- and dayworkers in the pulp and paper industry in 1952-2001. Scand J Work Environ Health. 2005;31(1):30-5.

6. Tenkanen L, Sjoblom T, Harma M. Joint effect of shift work and adverse life-style factors on the risk of coronary heart disease. Scand J Work Environ Health. 1998;24(5):351-7.

7. Guo Y, Rong Y, Huang X, Lai H, Luo X, Zhang Z, et al. Shift work and the relationship with metabolic syndrome in Chinese aged workers. PLoS One. 2015;10(3):e0120632.

8. Hansen J, Stevens RG. Case-control study of shift-work and breast cancer risk in Danish nurses: impact of shift systems. Eur J Cancer. 2012;48(11):1722-9.

9. Gu F, Han J, Laden F, Pan A, Caporaso NE, Stampfer MJ, et al. Total and cause-specific mortality of U.S. nurses working rotating night shifts. Am J Prev Med. 2015;48(3):241-52.

10. Fink AM. Measuring the effects of night-shift work on cardiac autonomic modulation: an appraisal of heart rate variability metrics. Int J Occup Med Environ Health. 2020;33(4):409-25.

11. Vyas MV, Garg AX, lansavichus AV, Costella J, Donner A, Laugsand LE, et al. Shift work and vascular events: systematic review and meta-analysis. BMJ. 2012;345:e4800.

12. Wang D, Ruan W, Chen Z, Peng Y, Li W. Shift work and risk of cardiovascular disease morbidity and mortality: a dose-response meta-analysis of cohort studies. Eur J Prev Cardiol. 2018;25(12):1293-302.

13. Torquati L, Mielke Gl, Brown WJ, Kolbe-Alexander T. Shift work and the risk of cardiovascular disease. A systematic review and meta-analysis including dose-response relationship. Scand J Work Environ Health. 2018:44(3):229-38.

14. Knutsson A. Health disorders of shift workers. Occup Med (Lond). 2003:53(2):103-8

15. Boggild H, Knutsson A. Shift work, risk factors and cardiovascular disease. Scand J Work Environ Health. 1999;25(2):85-99.

16. Evans JA, Davidson AJ. Health consequences of circadian disruption in humans and animal models. Prog Mol Biol Transl Sci. 2013;1 19:283-323.

17. Mohawk JA, Green CB, Takahashi JS. Central and peripheral circadian clocks in mammals. Annu Rev Neurosci. 2012;35:445-62.

18. Longo VD, Panda S. Fasting, circadian rhythms, and time-restricted feeding in healthy lifespan. Cell Metab. 2016;23(6):1048-59.

19. Mattson MP, Allison DB, Fontana L, Harvie M, Longo VD, Malaisse WJ, et al. Meal frequency and timing in health and disease. Proc Natl Acad Sci U S A. 2014:111(47):16647-53.

20. Salgado-Delgado R, Angeles-Castellanos M, Saderi N, Buijs RM, Escobar C. Food intake during the normal activity phase prevents obesity and circadian desynchrony in a rat model of night work. Endocrinology. 2010;151(3):1019-29.

21. Salgado-Delgado RC, Saderi N, Basualdo Mdel C, Guerrero-Vargas NN Escobar C, Buijs RM. Shift work or food intake during the rest phase promotes metabolic disruption and desynchrony of liver genes in male rats. PLoS One. 2013;8(4):e60052.

22. Salgado-Delgado R, Angeles-Castellanos M, Buijs MR, Escobar C. Internal desynchronization in a model of night-work by forced activity in rats. Neuroscience. 2008;154(3):922-31.

23. Weger BD, Gobet C, David FPA, Atger F, Martin E, Phillips NE, et al. Systematic analysis of differential rhythmic liver gene expression 
mediated by the circadian clock and feeding rhythms. Proc Natl Acad Sci U S A. 2021;118(3):e2015803118.

24. Kanehisa M, Furumichi M, Tanabe M, Sato Y, Morishima K. KEGG: new perspectives on genomes, pathways, diseases and drugs. Nucleic Acids Res. 2017;45(D1):D353-D61.

25. Nomura S, Satoh M, Fujita T, Higo T, Sumida T, Ko T, et al. Cardiomyocyte gene programs encoding morphological and functional signatures in cardiac hypertrophy and failure. Nat Commun. 2018;9(1):4435.

26. Hannan RD, Jenkins A, Jenkins AK, Brandenburger Y. Cardiac hypertrophy: a matter of translation. Clin Exp Pharmacol Physiol. 2003;30(8):517-27.

27. SiehI D, Chua BH, Lautensack-Belser N, Morgan HE. Faster protein and ribosome synthesis in thyroxine-induced hypertrophy of rat heart. Am J Physiol. 1985;248(3 Pt 1):C309-19.

28. Stamatoyannopoulos JA, Snyder M, Hardison R, Ren B, Gingeras T, Gilbert DM, et al. An encyclopedia of mouse DNA elements (Mouse ENCODE). Genome Biol. 2012;13(8):418.

29. Thurman RE, Rynes $E$, Humbert R, Vierstra J, Maurano MT, Haugen $E$, et al. The accessible chromatin landscape of the human genome. Nature. 2012:489(7414):75-82.

30. Yue F, Cheng Y, Breschi A, Vierstra J, Wu W, Ryba T, et al. A comparative encyclopedia of DNA elements in the mouse genome. Nature. 2014:515(7527):355-64.

31. Heinz S, Benner C, Spann N, Bertolino E, Lin YC, Laslo P, et al. Simple combinations of lineage-determining transcription factors prime cisregulatory elements required for macrophage and B cell identities. Mol Cell. 2010;38(4):576-89.

32. Hao G, Han Z, Meng Z, Wei J, Gao D, Zhang H, et al. Ets-1 upregulation mediates angiotensin II-related cardiac fibrosis. Int J Clin Exp Pathol. 2015;8(9):10216-27.

33. Zhan Y, Brown C, Maynard E, Anshelevich A, Ni W, Ho IC, et al. Ets-1 is a critical regulator of Ang II-mediated vascular inflammation and remodeling. J Clin Invest. 2005;115(9):2508-16.

34. Rommel C, Rosner S, Lother A, Barg M, Schwaderer M, Gilsbach R, et al. The transcription factor ETV1 induces atrial remodeling and arrhythmia. Circ Res. 2018;123(5):550-63.

35. Kelsey L, Flenniken AM, Qu D, Funnell AP, Pearson R, Zhou YQ, et al. ENU-induced mutation in the DNA-binding domain of KLF3 reveals important roles for KLF3 in cardiovascular development and function in mice. PLoS Genet. 2013:9(7):e1003612.

36. Sawaki D, Hou L, Tomida S, Sun J, Zhan H, Aizawa K, et al. Modulation of cardiac fibrosis by Kruppel-like factor 6 through transcriptional control of thrombospondin 4 in cardiomyocytes. Cardiovasc Res. 2015;107(4):420-30.

37. Jiang DS, Wei $X$, Zhang XF, Liu Y, Zhang Y, Chen $K$, et al. IRF8 suppresses pathological cardiac remodelling by inhibiting calcineurin signalling. Nat Commun. 2014:5:3303.

38. Bouveret R, Waardenberg AJ, Schonrock N, Ramialison M, Doan T, de Jong D, et al. NKX2-5 mutations causative for congenital heart disease retain functionality and are directed to hundreds of targets. Elife. 2015;4.

39. Shekhar A, Lin X, Liu FY, Zhang J, Mo H, Bastarache L, et al. Transcription factor ETV1 is essential for rapid conduction in the heart. J Clin Invest. 2016:126(12):4444-59.

40. Kalna V, Yang Y, Peghaire CR, Frudd K, Hannah R, Shah AV, et al. The transcription factor ERG regulates super-enhancers associated with an endothelial-specific gene expression program. Circ Res. 2019:124(9):1337-49

41. Keenan JE, Sulliman H, Ulrich A, Piantadosi CA. Cardiomyocyte-specific ablation of nuclear respiratory factor 1 in the mouse leads to dysregulation of mitochondrial biogenesis, apoptosis, and heart failure. Circ Res. 2014;115(suppl_1):A86.

42. Sweet ME, Cocciolo A, Slavov D, Jones KL, Sweet JR, Graw SL, et al. Transcriptome analysis of human heart failure reveals dysregulated cell adhesion in dilated cardiomyopathy and activated immune pathways in ischemic heart failure. BMC Genomics. 2018;19(1):812.

43. Xia C, Braunstein Z, Toomey AC, Zhong J, Rao X. S100 proteins as an important regulator of macrophage inflammation. Front Immunol. 2017:8:1908.

44. Schneider M, Kostin S, Strom CC, Aplin M, Lyngbaek S, Theilade J, et al. S100A4 is upregulated in injured myocardium and promotes growth and survival of cardiac myocytes. Cardiovasc Res. 2007;75(1):40-50.
45. Troncoso R, Ibarra C, Vicencio JM, Jaimovich E, Lavandero S. New insights into IGF-1 signaling in the heart. Trends Endocrinol Metab. 2014:25(3):128-37.

46. Hua Y, Robinson TJ, Cao Y, Shi GP, Ren J, Nair S. Cathepsin K knockout alleviates aging-induced cardiac dysfunction. Aging Cell. 2015;14(3):345-51.

47. De Mello WC, Danser AH. Angiotensin II and the heart : on the intracrine renin-angiotensin system. Hypertension. 2000;35(6):1183-8.

48. Chisalita SI, Dahlstrom U, Arnqvist HJ, Alehagen U. Increased IGF1 levels in relation to heart failure and cardiovascular mortality in an elderly population: impact of ACE inhibitors. Eur J Endocrinol. 2011;165(6):891-8.

49. Altin JG, Sloan EK. The role of CD45 and CD45-associated molecules in T cell activation. Immunol Cell Biol. 1997;75(5):430-45.

50. Chistiakov DA, Killingsworth MC, Myasoedova VA, Orekhov AN, Bobryshev YV. CD68/macrosialin: not just a histochemical marker. Lab Invest. 2017:97(1):4-13.

51. Salgado-Delgado R, Nadia S, Angeles-Castellanos M, Buijs RM, Escobar C. In a rat model of night work, activity during the normal resting phase produces desynchrony in the hypothalamus. J Biol Rhythms. 2010;25(6):421-31.

52. Manella G, Sabath E, Aviram R, Dandavate V, Ezagouri S, Golik M, et al. The liver-clock coordinates rhythmicity of peripheral tissues in response to feeding. Nat Metab. 2021;3(6):829-42.

53. Archer SN, Oster H. How sleep and wakefulness influence circadian rhythmicity: effects of insufficient and mistimed sleep on the animal and human transcriptome. J Sleep Res. 2015;24(5):476-93.

54. Wolff G, Esser KA. Scheduled exercise phase shifts the circadian clock in skeletal muscle. Med Sci Sports Exerc. 2012;44(9):1663-70.

55. Kemler D, Wolff CA, Esser KA. Time-of-day dependent effects of contractile activity on the phase of the skeletal muscle clock. J Physiol. 2020;598(17):3631-44.

56. Greenwell BJ, Trott AJ, Beytebiere JR, Pao S, Bosley A, Beach E, et al. Rhythmic food intake drives rhythmic gene expression more potently than the hepatic circadian clock in mice. Cell reports. 2019;27(3):649-57 e5.

57. Atger F, Gobet C, Marquis J, Martin E, Wang J, Weger B, et al. Circadian and feeding rhythms differentially affect rhythmic mRNA transcription and translation in mouse liver. Proc Natl Acad Sci U S A. 2015;112(47):E6579-88.

58. Guan $D$, Xiong $Y$, Trinh TM, Xiao Y, Hu W, Jiang C, et al. The hepatocyte clock and feeding control chronophysiology of multiple liver cell types. Science. 2020;369(6509):1388-94.

59. Palm W, Thompson CB. Nutrient acquisition strategies of mammalian cells. Nature. 2017;546(7657):234-42.

60. Nguyen KD, Fentress SJ, Qiu Y, Yun K, Cox JS, Chawla A. Circadian gene Bmal1 regulates diurnal oscillations of Ly6C(hi) inflammatory monocytes. Science. 2013;341(6153):1483-8.

61. Charkoudian N, Rabbitts JA. Sympathetic neural mechanisms in human cardiovascular health and disease. Mayo Clin Proc. 2009;84(9):822-30.

62. Sharrocks AD. The ETS-domain transcription factor family. Nat Rev Mol Cell Biol. 2001;2(11):827-37.

63. Selvaraj N, Kedage V, Hollenhorst PC. Comparison of MAPK specificity across the ETS transcription factor family identifies a high-affinity ERK interaction required for ERG function in prostate cells. Cell Commun Signal. 2015;13:12.

64. Brandenburger Y, Arthur JF, Woodcock EA, Du XJ, Gao XM, Autelitano $D J$, et al. Cardiac hypertrophy in vivo is associated with increased expression of the ribosomal gene transcription factor UBF. FEBS Lett. 2003;548(1-3):79-84.

65. Bonnans $C$, Chou J, Werb Z. Remodelling the extracellular matrix in development and disease. Nat Rev Mol Cell Biol. 2014;15(12):786-801.

66. Gurtl B, Kratky D, Guelly C, Zhang L, Gorkiewicz G, Das SK, Tamilarasan KP, et al. Apoptosis and fibrosis are early features of heart failure in an animal model of metabolic cardiomyopathy. 2009 (1365-2613 (Electronic)).

67. Vogel B, Siebert H, Hofmann U, Frantz S. Determination of collagen content within picrosirius red stained paraffin-embedded tissue sections using fluorescence microscopy. MethodsX. 2015;2:124-34.

68. Khalil H, Kanisicak O, Prasad V, Correll RN, Fu X, Schips T, et al. Fibroblastspecific TGF-beta-Smad2/3 signaling underlies cardiac fibrosis. J Clin Invest. 2017;127(10):3770-83.

69. Ali SR, Ranjbarvaziri S, Talkhabi M, Zhao P, Subat A, Hojjat A, et al. Developmental heterogeneity of cardiac fibroblasts does not predict pathological proliferation and activation. Circ Res. 2014;115(7):625-35. 
70. Conway SJ, Molkentin JD. Periostin as a heterofunctional regulator of cardiac development and disease. Curr Genomics. 2008;9(8):548-55.

71. Zhang CL, Zhao Q, Liang H, Qiao X, Wang JY, Wu D, et al. Cartilage intermediate layer protein-1 alleviates pressure overload-induced cardiac fibrosis via interfering TGF-beta1 signaling. J Mol Cell Cardiol. 2018;116:135-44.

72. Kuusisto J, Karja V, Sipola P, Kholova I, Peuhkurinen K, Jaaskelainen P, et a Low-grade inflammation and the phenotypic expression of myocardial fibrosis in hypertrophic cardiomyopathy. Heart. 2012;98(13):1007-13.

73. Duerr GD, Heinemann JC, Dunkel S, Zimmer A, Lutz B, Lerner R, et al. Myocardial hypertrophy is associated with inflammation and activation of endocannabinoid system in patients with aortic valve stenosis. Life Sci. 2013;92(20-21):976-83.

74. Hatori M, Vollmers C, Zarrinpar A, DiTacchio L, Bushong EA, Gill S, et al. Time-restricted feeding without reducing caloric intake prevents metabolic diseases in mice fed a high-fat diet. Cell Metab. 2012;15(6):848-60.

75. Chaix A, Zarrinpar A, Miu P, Panda S. Time-restricted feeding is a preventative and therapeutic intervention against diverse nutritional challenges. Cell Metab. 2014;20(6):991-1005

76. Wilkinson MJ, Manoogian ENC, Zadourian A, Lo H, Fakhouri S, Shoghi A, et al. Ten-hour time-restricted eating reduces weight, blood pressure, and atherogenic lipids in patients with metabolic syndrome. Cell Metab. 2020;31(1):92-104 e5.

77. Regmi P, Heilbronn LK. Time-restricted eating: benefits, mechanisms, and challenges in translation. iscience. 2020;23(6):101161.

78. Kim D, Pertea G, Trapnell C, Pimentel H, Kelley R, Salzberg SL. TopHat2: accurate alignment of transcriptomes in the presence of insertions, deletions and gene fusions. Genome Biol. 2013;14(4):R36.

79. Trapnell C, Roberts A, Goff L, Pertea G, Kim D Kelley DR, Pimentel H, et al. Differential gene and transcript expression analysis of RNA-seq experiments with TopHat and Cufflinks. 2012 (1750-2799 (Electronic)).

80. Thorvaldsdóttir H, Robinson JT, Mesirov JP. Integrative Genomics Viewer (IGV): high-performance genomics data visualization and exploration. Brief Bioinformatics. 2013;14(2):178-92.

81. Langmead B, Salzberg SL. Fast gapped-read alignment with Bowtie 2. Nat Methods. 2012;9(4):357-9.

82. Li H, Handsaker B, Wysoker A, Fennell T, Ruan J, Homer N, et al. The sequence alignment/map format and SAMtools. Bioinformatics (Oxford, England). 2009;25(16):2078-9.

83. Quinlan AR, Hall IM. BEDTools: a flexible suite of utilities for comparing genomic features. Bioinformatics (Oxford, England). 2010;26(10):841-2.

84. Wijnen $\mathrm{H}$, Naef F, Young MW. Molecular and statistical tools for circadian transcript profiling. Methods Enzymol. 2005;393:341-65.

85. Hutchison AL, Maienschein-Cline M, Chiang AH, Tabei SM, Gudjonson H, Bahroos N, et al. Improved statistical methods enable greater sensitivity in rhythm detection for genome-wide data. PLoS Comput Biol. 2015;11(3):e1004094

86. Wu G, Anafi RC, Hughes ME, Kornacker K, Hogenesch JB. MetaCycle: an integrated $\mathrm{R}$ package to evaluate periodicity in large scale data. Bioinformatics. 2016;32(21):3351-3.

87. Hughes ME, DiTacchio L, Hayes KR, Vollmers C, Pulivarthy S, Baggs JE, et al. Harmonics of circadian gene transcription in mammals. PLoS Genet. 2009:5(4):e1000442.

88. Thaben PF, Westermark PO. Detecting rhythms in time series with RAIN. J Biol Rhythms. 2014;29(6):391-400

89. Benjamini $Y$, Hochberg $Y$. Controlling the false discovery rate: a practical and powerful approach to multiple testing. J R Stat Soc Ser B (Methodological). 1995;57(1):289-300.

90. Ritchie ME, Phipson B, Wu D, Hu Y, Law CW, Shi W, et al. limma powers differential expression analyses for RNA-sequencing and microarray studies. Nucleic Acids Res. 2015;43(7):e47.

91. Pagès H, Falcon S, Carlson M, Li N. AnnotationDbi: manipulation of SQLite-based annotations in Bioconductor. R package version 1480; 2019.

92. Huang S, Yeo AA, Li SD. Modification of Kolmogorov-Smirnov test for DNA content data analysis through distribution alignment. Assay Drug Dev Technol. 2007:5(5):663-71.

93. Pilling D, Roife D, Wang M, Ronkainen SD, Crawford JR, Travis EL, et al. Reduction of bleomycin-induced pulmonary fibrosis by serum amyloid $P$. J Immunol. 2007;179(6):4035-44.
94. Karhadkar TR, Meek TD, Gomer RH. Inhibiting sialidase-induced TGFbeta 1 activation attenuates pulmonary fibrosis in mice. J Pharmaco Exp Ther. 2021:376(1):106-17.

\section{Publisher's Note}

Springer Nature remains neutral with regard to jurisdictional claims in published maps and institutional affiliations.
Ready to submit your research? Choose BMC and benefit from:

- fast, convenient online submission

- thorough peer review by experienced researchers in your field

- rapid publication on acceptance

- support for research data, including large and complex data types

- gold Open Access which fosters wider collaboration and increased citations

- maximum visibility for your research: over $100 \mathrm{M}$ website views per year

At BMC, research is always in progress.

Learn more biomedcentral.com/submissions 